REPRESENTATION THEORY

An Electronic Journal of the American Mathematical Society

Volume 5, Pages 284-316 (October 3, 2001)

S $1088-4165(01) 00124-8$

\title{
THE CLOSURE DIAGRAM FOR NILPOTENT ORBITS OF THE SPLIT REAL FORM OF $E_{7}$
}

\author{
DRAGOMIR Ž. ĐOKOVIĆ
}

\begin{abstract}
Let $\mathcal{O}_{1}$ and $\mathcal{O}_{2}$ be adjoint nilpotent orbits in a real semisimple Lie algebra. Write $\mathcal{O}_{1} \geq \mathcal{O}_{2}$ if $\mathcal{O}_{2}$ is contained in the closure of $\mathcal{O}_{1}$. This defines a partial order on the set of such orbits, known as the closure ordering. We determine this order for the split real form $\mathrm{EV}$ of $E_{7}$.
\end{abstract}

\section{INTRODUCTION}

The closure diagrams for adjoint nilpotent orbits in noncompact real forms of the exceptional simple complex Lie algebras were determined in [8, 9, 10, 11, except for the split real forms of $E_{7}$ and $E_{8}$. Here we handle the split real form of $E_{7}$ (also known as the form of type EV, in Cartan's notation). This paper is essentially a continuation of our paper [10 and we shall often refer to it.

By $\mathfrak{g}$ we denote a simple complex Lie algebra of type $E_{7}$, by $\mathfrak{g}_{0}$ a split real form of $\mathfrak{g}$, and by $G$ (respectively $G_{0}$ ) the adjoint group of $\mathfrak{g}$ (respectively $\mathfrak{g}_{0}$ ). As usual, let $\mathfrak{g}_{0}=\mathfrak{k}_{0} \oplus \mathfrak{p}_{0}$ be a Cartan decomposition of $\mathfrak{g}_{0}, \mathfrak{g}=\mathfrak{k} \oplus \mathfrak{p}$ its complexification, and $\theta$ the Cartan involution. Let $\sigma$ be the complex conjugation of $\mathfrak{g}$ with respect to $\mathfrak{g}_{0}$, and let $\mathfrak{h}$ be a $\sigma$-stable Cartan subalgebra of $\mathfrak{k}$. Since $\mathfrak{g}_{0}$ is of inner type, $\mathfrak{h}$ is also a Cartan subalgebra of $\mathfrak{g}$.

Denote by $\mathcal{N}$ the nilpotent variety of $\mathfrak{g}$ and set

$$
\mathcal{N}_{\mathbf{R}}=\mathcal{N} \cap \mathfrak{g}_{0}, \quad \mathcal{N}_{1}=\mathcal{N} \cap \mathfrak{p} .
$$

Let $K^{0} \cong \mathrm{SL}_{8} / Z_{4}$ be the connected subgroup of $G$ with the Lie algebra k. (By $Z_{k}$ we denote a cyclic group of order $k$.) It is known that the orbit spaces $\mathcal{N}_{\mathbf{R}} / G_{0}$ and $\mathcal{N}_{1} / K^{0}$, equipped with the quotient topologies, are homeomorphic and that the Kostant-Sekiguchi bijection is a homeomorphism $\mathcal{N}_{\mathbf{R}} / G_{0} \rightarrow \mathcal{N}_{1} / K^{0}$ (see [5, 1]). Our main result, Theorem 4.1, describes the topology of $\mathcal{N}_{1} / K^{0}$. The description is via the closure diagram shown in Figures 5 and 6 . The construction of this diagram and the proof of its correctness are based on extensive and nontrivial computations for which we used heavily Maple [4] and LiE [16] (in addition to our own programs). It may be worthwhile to point out that Table 3 contains an extensive list of the representatives of all nonzero nilpotent $K^{0}$-orbits in $\mathfrak{p}$. In many cases several representatives of different types are given.

It is a pleasure to thank J. Sekiguchi for his interest and several discussions that we had about the closures of nilpotent orbits. The proposition of Section 7 is in fact a by-product of these discussions.

Received by the editors March 9, 2001 and, in revised form, August 17, 2001.

2000 Mathematics Subject Classification. Primary 05B15, 05B20; Secondary 05B05.

Supported in part by the NSERC Grant A-5285. 


\section{Preliminaries}

The closure diagram for adjoint nilpotent orbits in $\mathfrak{g}$ was determined by Mizuno 12] and verified later by Beynon and Spaltenstein [2]. We will use the version of Mizuno's diagram given in [10. Figure 1] where each node represents a $G$-orbit in $\mathcal{N}$ and is labeled by the corresponding Bala-Carter symbol (see [5]). In this diagram, the orbits having the same dimension are positioned at the same level. Because of its length, the diagram is split into two pieces. The dimensions of the orbits are indicated on both sides of the figure.

There are 45 adjoint nilpotent orbits in $\mathfrak{g}$ (including the trivial orbit). The nonzero ones are listed in [10, Table 1]. The $k$-th orbit, i.e., the one that appears as the $k$-th entry of that table, will be denoted by $\mathcal{O}^{k}$. The second column of that table contains the Bala-Carter symbol of $\mathcal{O}^{k}$, and the third one gives the weighted Dynkin diagram of $\mathcal{O}^{k}$. The complex dimension of $\mathcal{O}^{k}$ is recorded in the last column.

The nonzero $G_{0}$-orbits in $\mathcal{N}_{\mathbf{R}}$, or equivalently the nonzero $K^{0}$-orbits in $\mathcal{N}_{1}$, were classified in [6] (see also [5]). We shall keep the same numbering for these orbits as in these two references. The $i$-th nontrivial $G_{0}$-orbit in $\mathcal{N}_{\mathbf{R}}$ will be denoted by $\mathcal{O}_{0}^{i}$, and we denote by $\mathcal{O}_{1}^{i}$ the nontrivial $K^{0}$-orbit in $\mathcal{N}_{1}$ that corresponds to $\mathcal{O}_{0}^{i}$ under the Kostant-Sekiguchi bijection. The fourth column of [10, Table 1] gives the superscripts $i$ of the orbits $\mathcal{O}_{0}^{i}$ (or, equivalently, $\mathcal{O}_{1}^{i}$ ) which are contained in $\mathcal{O}^{k}$. For instance, if $k=7$, then

$$
\mathcal{O}^{7} \cap \mathfrak{g}_{0}=\mathcal{O}_{0}^{10} \cup \mathcal{O}_{0}^{11} \cup \mathcal{O}_{0}^{12}, \quad \mathcal{O}^{7} \cap \mathfrak{p}=\mathcal{O}_{1}^{10} \cup \mathcal{O}_{1}^{11} \cup \mathcal{O}_{1}^{12} .
$$

We say that a triple $(E, H, F)$ in $\mathfrak{g}$ is a standard triple if $[H, E]=2 E,[H, F]=$ $-2 F,[F, E]=H$ and $E, H, F$ are nonzero. Such a triple is normal if also $H \in \mathfrak{k}$ and $E, F \in \mathfrak{p}$. We denote the root system of $(\mathfrak{g}, \mathfrak{h})$ by $R$, choose a system of positive roots $R^{+} \subset R$ and a base $B=\left\{\alpha_{i}: 1 \leq i \leq 7\right\} \subset R^{+}$of $R$. The simple roots $\alpha_{i} \in B$ are indexed as in Figure 1 .

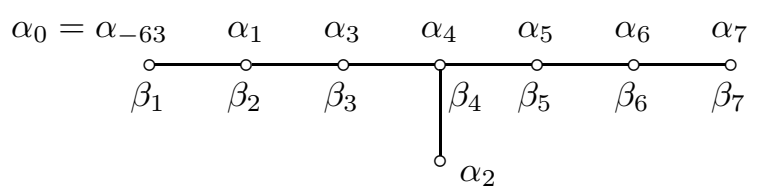

Figure 1 . The extended base of $E_{7}$ and a base of $R_{0}$

Let us also introduce the subgroup $K=\{x \in G: \theta(x)=x\}$. Its identity component is the group $K^{0}$ defined above and $K / K^{0}=Z_{2}$.

We extend the enumeration of simple roots $\alpha_{i}, 1 \leq i \leq 7$, to the enumeration $\alpha_{i}, 1 \leq i \leq 63$, of $R^{+}$. It is given in [10, Appendix]. A negative root $-\alpha_{i}$ will be also written as $\alpha_{-i}$. The coroot of $\alpha_{i}$ is denoted by $H_{i} \in \mathfrak{h}$. For $\alpha \in R$ we let $\mathfrak{g}^{\alpha}$ be the root space of $\alpha$. A nonzero element $X_{\alpha} \in \mathfrak{g}^{\alpha}$ is called a root vector of $\alpha$. We assume that a root vector $X_{i}$ has been chosen for each root $\alpha_{i}, \pm i \in\{1, \ldots, 63\}$.

By recalling the definition of the $Z_{2}$-gradation $\mathfrak{g}=\mathfrak{k}+\mathfrak{p}$ from [6], one can easily distinguish the roots $\alpha$ for which $\mathfrak{g}^{\alpha} \subset \mathfrak{k}$ from those for which $\mathfrak{g}^{\alpha} \subset \mathfrak{p}$. Indeed, if $\alpha=k_{1} \alpha_{1}+k_{2} \alpha_{2}+\cdots+k_{7} \alpha_{7}$, then in the former case $k_{2}$ is even $\left(k_{2}=0, \pm 2\right)$ and in the latter it is odd $\left(k_{2}= \pm 1\right)$. 
By adjoining the negative of the highest root $\alpha_{0}=-\alpha_{63}=\alpha_{-63}$ to $B$ we obtain the so-called extended base $B_{e}=B \cup\left\{\alpha_{0}\right\}$ (see Figure 1). Let $R_{0}$ be the root system of $(\mathfrak{k}, \mathfrak{h})$ where we view $R_{0}$ as a subsystem of $R$. We set $R_{0}^{+}=R_{0} \cap R^{+}$and denote by $B_{0}$ the unique base of $R_{0}$ contained in $R_{0}^{+}$. It turns out that $B_{0} \subset B_{e}$. Explicitly we have

$$
B_{0}=\left\{\alpha_{0}, \alpha_{1}, \alpha_{3}, \alpha_{4}, \alpha_{5}, \alpha_{6}, \alpha_{7}\right\}=\left\{\beta_{i}: 1 \leq i \leq 7\right\},
$$

where, as in [6, Table IV],

$$
\beta_{1}=\alpha_{0}, \beta_{2}=\alpha_{1}, \beta_{3}=\alpha_{3}, \beta_{4}=\alpha_{4}, \beta_{5}=\alpha_{5}, \beta_{6}=\alpha_{6}, \beta_{7}=\alpha_{7} .
$$

Given a nonzero $K^{0}$-orbit $\mathcal{O}_{1}^{i} \subset \mathcal{N}_{1}$, we can choose a normal triple $(E, H, F)$ such that $E \in \mathcal{O}_{1}^{i}, H \in \mathfrak{h}$, and $\beta_{i}(H) \geq 0$ for $1 \leq i \leq 7$. The integers $\beta_{i}(H)$ determine uniquely $H$ and, consequently, also the orbit $\mathcal{O}_{1}^{i}$.

Let $s_{i}$ be the reflection corresponding to the root $\alpha_{i}$. The roots $\alpha_{47}, \alpha_{48}$, and $\alpha_{49}$ are mutually orthogonal. The element $w=s_{47} s_{48} s_{49}$ of the Weyl group $W=$ $W(\mathfrak{g}, \mathfrak{h})$ is an involution. We have $w\left(B_{0}\right)=B_{0}$ and $w$ induces the automorphism of $B_{0}$ of order 2, i.e., $w\left(\beta_{i}\right)=\beta_{8-i}$ for $1 \leq i \leq 7$. There is an involution $\tilde{w} \in G$ (a lifting of $w$ ) such that $\tilde{w}(\mathfrak{h})=\mathfrak{h}$ and the action of $\tilde{w}$ on the dual space $\mathfrak{h}^{*}$ coincides with the action of $w$.

We remark that the standard regular subalgebra (see [10] for the definition) with simple roots $\alpha_{47}, \alpha_{48}$, and $\alpha_{49}$ is of type $\left(3 A_{1}\right)^{\prime \prime}$. Indeed if we set

$$
\gamma_{1}=\alpha_{-2}, \gamma_{2}=\alpha_{47}, \gamma_{3}=\alpha_{-17}, \gamma_{4}=\alpha_{-44}, \gamma_{5}=\alpha_{48}, \gamma_{6}=\alpha_{-19}, \gamma_{7}=\alpha_{49},
$$

then these roots form another base of $R$ and so there is an element of $W$ that maps $\gamma_{i} \rightarrow \alpha_{i}$ for $1 \leq i \leq 7$. Hence the triple $\left\{\alpha_{47}, \alpha_{48}, \alpha_{49}\right\}$ is mapped onto the triple $\left\{\alpha_{2}, \alpha_{5}, \alpha_{7}\right\}$ which is a base of a system of type $\left(3 A_{1}\right)^{\prime \prime}$.

Let us briefly indicate how the element $\tilde{w}$ can be explicitly constructed. For that purpose only, we shall assume that the root vectors $X_{i}$ for $\pm i \in\{1, \ldots, 63\}$ have been chosen (say as in [7]) so that together with the $H_{i}, 1 \leq i \leq 7$, they form a Chevalley basis of $\mathfrak{g}_{0}$. In particular, $\left[X_{-i}, X_{i}\right]=H_{i}$ for all indices $\pm i \in\{1, \ldots, 63\}$. For $\alpha \in R$ let

$$
\theta_{\alpha}=e^{\operatorname{ad}\left(X_{\alpha}\right)} e^{\operatorname{ad}\left(X_{-\alpha}\right)} e^{\operatorname{ad}\left(X_{\alpha}\right)},
$$

and set $\theta_{i}=\theta_{\alpha_{i}}($ see [3]). Then we can take

$$
\tilde{w}=\theta_{47} \theta_{48} \theta_{49} .
$$

Since the roots $\alpha_{47}, \alpha_{48}$, and $\alpha_{49}$ are mutually orthogonal, we have

$$
\tilde{w}^{2}=\theta_{47}^{2} \theta_{48}^{2} \theta_{49}^{2} .
$$

This element is the identity because $G$ is the adjoint group and $\left\{\alpha_{47}, \alpha_{48}, \alpha_{49}\right\}$ is a base of a system of type $\left(3 A_{1}\right)^{\prime \prime}$. The analogously defined element in the simply connected group of type $E_{7}$ is the central involution of that group (for more details see [15]).

Since $w\left(B_{0}\right)=B_{0}$, we have $\tilde{w}(\mathfrak{k})=\mathfrak{k}$ and $\tilde{w}(\mathfrak{p})=\mathfrak{p}$. As $\tilde{w}$ induces an outer automorphism of $\mathfrak{k}$, we conclude that $\tilde{w}$ belongs to the non-identity component of $K$. The action of $w$ (and $\tilde{w}$ ) on $R$ is described in Table 1. The involution $\tilde{w}$ permutes the nilpotent $K^{0}$-orbits in $\mathfrak{p}$ and induces a homeomorphism of the orbit space $\mathcal{N}_{1} / K^{0}$. This permutation action is given in Table 2 . The fact that $\tilde{w}$ induces an involutorial automorphism of the closure diagram will be used to simplify the proof of our main result. 
TABLE 1 . The pairs $i, j$ such that $w\left(\alpha_{i}\right)=\alpha_{j}$

\begin{tabular}{|rr|rr|rr|rr|rr|}
\hline 1 & 6 & 2 & 2 & 3 & 5 & 4 & 4 & 7 & -63 \\
8 & 12 & 9 & 9 & 10 & 11 & 13 & -62 & 14 & 18 \\
15 & 16 & 17 & 17 & 19 & -61 & 20 & 23 & 21 & 24 \\
22 & 22 & 25 & -60 & 26 & 29 & 27 & 27 & 28 & 28 \\
30 & -59 & 31 & -58 & 32 & 35 & 33 & 33 & 34 & -56 \\
36 & -57 & 37 & 40 & 38 & 38 & 39 & -54 & 41 & -55 \\
42 & 43 & 44 & -51 & 45 & -52 & 46 & 46 & 47 & -47 \\
48 & -48 & 49 & -49 & 50 & 50 & 51 & -44 & 52 & -45 \\
53 & 53 & 54 & -39 & 55 & -41 & 56 & -34 & 57 & -36 \\
58 & -31 & 59 & -30 & 60 & -25 & 61 & -19 & 62 & -13 \\
63 & -7 & -1 & -6 & -2 & -2 & -3 & -5 & -4 & -4 \\
-8 & -12 & -9 & -9 & -10 & -11 & -14 & -18 & -15 & -16 \\
-17 & -17 & -20 & -23 & -21 & -24 & -22 & -22 & -26 & -29 \\
-27 & -27 & -28 & -28 & -32 & -35 & -33 & -33 & -37 & -40 \\
-38 & -38 & -42 & -43 & -46 & -46 & -50 & -50 & -53 & -53 \\
\hline
\end{tabular}

TABLE 2. The pairs $i, j$ such that $\tilde{w}\left(\mathcal{O}_{1}^{i}\right)=\mathcal{O}_{1}^{j}$

\begin{tabular}{|rr|rr|rr|rr|rr|rr|rr|rr|}
\hline 1 & 1 & 2 & 2 & 3 & 4 & 5 & 5 & 6 & 6 & 7 & 7 & 8 & 9 & 10 & 11 \\
12 & 12 & 13 & 14 & 15 & 15 & 16 & 17 & 18 & 19 & 20 & 20 & 21 & 21 & 22 & 23 \\
24 & 24 & 25 & 25 & 26 & 26 & 27 & 27 & 28 & 29 & 30 & 30 & 31 & 32 & 33 & 34 \\
35 & 36 & 37 & 37 & 38 & 38 & 39 & 40 & 41 & 42 & 43 & 43 & 44 & 45 & 46 & 47 \\
48 & 49 & 50 & 50 & 51 & 52 & 53 & 53 & 54 & 54 & 55 & 56 & 57 & 58 & 59 & 59 \\
60 & 61 & 62 & 62 & 63 & 63 & 64 & 65 & 66 & 66 & 67 & 68 & 69 & 70 & 71 & 71 \\
72 & 73 & 74 & 75 & 76 & 77 & 78 & 79 & 80 & 80 & 81 & 81 & 82 & 83 & 84 & 84 \\
85 & 86 & 87 & 88 & 89 & 90 & 91 & 92 & 93 & 94 & & & & & & \\
\hline
\end{tabular}

The basic technique for the construction of the closure diagrams of nilpotent orbits was described in [8] and perfected later in our subsequent papers [9, 10, 11. It is especially convenient for real forms of inner type and will be employed in this paper.

We introduce some more notation concerning a normal triple $(E, H, F)$. For any integer $j$ we define the subspaces

$$
\begin{aligned}
& \mathfrak{g}_{H}(0, j)=\{X \in \mathfrak{k}:[H, X]=j X\}, \\
& \mathfrak{g}_{H}(1, j)=\{X \in \mathfrak{p}:[H, X]=j X\},
\end{aligned}
$$

and for integers $i \geq 1$ we set

$$
\mathfrak{p}_{i}(H)=\sum_{j \geq i} \mathfrak{g}_{H}(1, j)
$$

By $Q_{H}$ we denote the parabolic subgroup of $K^{0}$ with Lie algebra

$$
\mathfrak{q}_{H}=\sum_{j \geq 0} \mathfrak{g}_{H}(0, j) .
$$




\section{Representatives of the nOnZero nilpotent $K^{0}$-orbits in $\mathfrak{p}$}

There are exactly 95 nilpotent $K^{0}$-orbits in $\mathfrak{p}$. They are denoted by $\mathcal{O}_{1}^{i}, 0 \leq i \leq$ 94 , where $\mathcal{O}_{1}^{0}=\{0\}$ is the trivial orbit. Thus

$$
\mathcal{N}_{1}=\bigcup_{i=0}^{94} \mathcal{O}_{1}^{i}
$$

The 94 nontrivial nilpotent $K^{0}$-orbits $\mathcal{O}_{1}^{i}$ in $\mathfrak{p}$ and their representatives $E^{i}$ are listed in Table 3. (While writing [6], we compiled the lists of representatives of the nilpotent $K^{0}$-orbits in $\mathfrak{p}$, but they were not included in that paper.)

For each $i \in\{1, \ldots, 94\}$ we record in the first column the integer $k$ such that $\mathcal{O}_{1}^{i} \subset \mathcal{O}^{k}$ (see also [10, Table 1]). We choose a normal triple $\left(E^{i}, H^{i}, F^{i}\right)$ with $E^{i} \in \mathcal{O}_{1}^{i}, H^{i} \in \mathfrak{h}$, and such that $\beta_{j}\left(H^{i}\right) \geq 0,1 \leq j \leq 7$. The third column lists the integers $\beta_{j}\left(H^{i}\right)$. The last two columns give a representative $E^{i} \in \mathcal{O}_{1}^{i}$ and its type (see below for more details about types). The representative $E^{i}$ is written as a sum of root vectors all of which lie in the subspace $\mathfrak{g}_{H^{i}}(1,2)$ (this can be verified by using Table 7 given in the Appendix). The corresponding roots are always linearly independent. In many cases we give several representatives of different types.

Let $i<j$ be such that $\tilde{w}\left(\mathcal{O}_{1}^{i}\right)=\mathcal{O}_{1}^{j}$ (see Table 2). Such pairs are recognizable from Table 3 because they satisfy

$$
\beta_{k}\left(H^{j}\right)=\beta_{8-k}\left(H^{i}\right), \quad 1 \leq k \leq 7 .
$$

Our representatives for $\mathcal{O}_{1}^{j}$ are obtained from those of $\mathcal{O}_{1}^{i}$ by applying the involution $w$. For instance if $i=3$ and $j=4$, then $E^{3}=\left(X_{-2}\right)+\left(X_{-28}\right)+\left(X_{-49}\right)$ is our representative of $\mathcal{O}_{1}^{3}$. Since $w$ maps the roots $\alpha_{-2}, \alpha_{-28}$, and $\alpha_{-49}$ to the roots $\alpha_{-2}, \alpha_{-28}$, and $\alpha_{49}$ (see Table 1 ), we use $E^{4}=\left(X_{-2}\right)+\left(X_{-28}\right)+\left(X_{49}\right)$ as our representative of the orbit $\mathcal{O}_{1}^{4}$.

If the type of $E^{i}$ is written as a sum of two or more symbols, then we enclose in parentheses the contribution of each of these symbols. For instance the representative

$$
E^{46}=\left(X_{-32}+X_{50}+X_{-40}+X_{-42}\right)+\left(X_{-41}\right)
$$

of $\mathcal{O}_{1}^{46}$ is of type $D_{4}+A_{1}$. The contribution of $D_{4}$ (resp. $A_{1}$ ) is $X_{-32}+X_{50}+$ $X_{-40}+X_{-42}$ (resp. $\left.X_{-41}\right)$. The roots $\alpha_{-32}, \alpha_{50}, \alpha_{-40}, \alpha_{-42}$, and $\alpha_{-41}$ form a base of a root subsystem of type $D_{4}+A_{1}$ (see Figure 2).

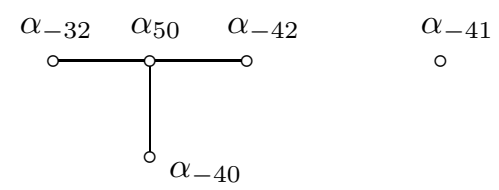

Figure 2. A base of a subsystem of type $D_{4}+A_{1}$

In Figures 3 and 4 we give the Dynkin-like diagrams for the non-standard types that occur in Tables 3 and 4 . The dotted lines in these diagrams join two roots whose angle is $\pi / 3$ while the solid lines (as usual) join two roots whose angle is 
$2 \pi / 3$. More precisely one should view these diagrams as the objects defining the types of representatives. For the type $E_{7}\left(a_{4}\right)$ see Figure 7 .
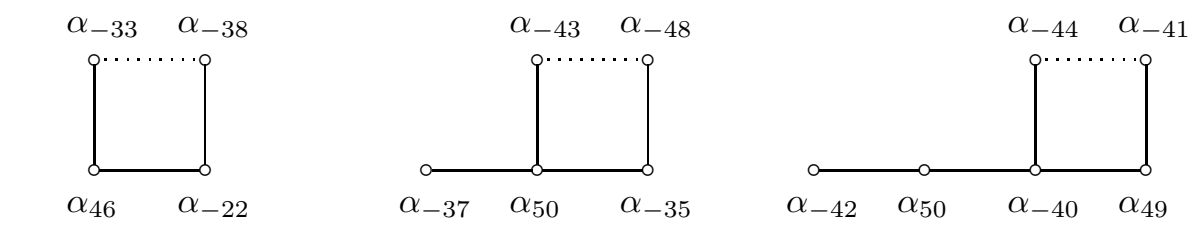

$D_{4}\left(a_{1}\right)$ : orbit 27

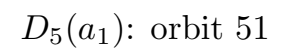

$D_{6}\left(a_{1}\right)$ : orbit 72
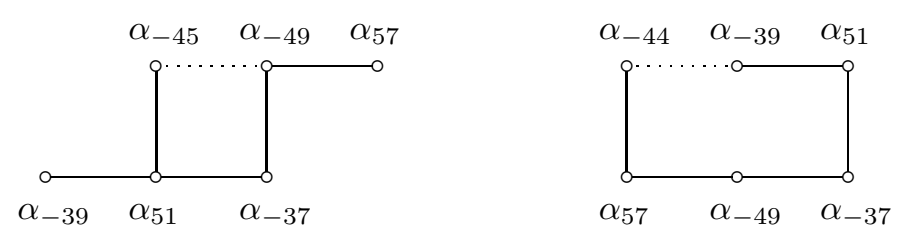

$D_{6}\left(a_{2}\right):$ orbit 64
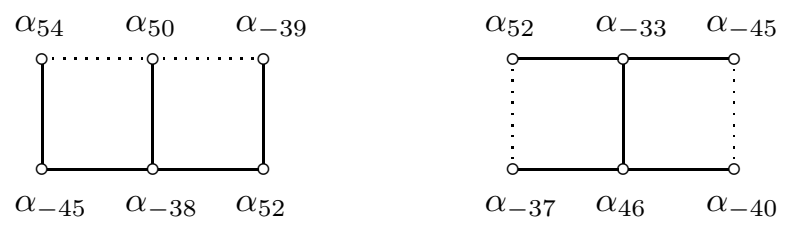

$E_{6}\left(a_{3}\right):$ orbit 63
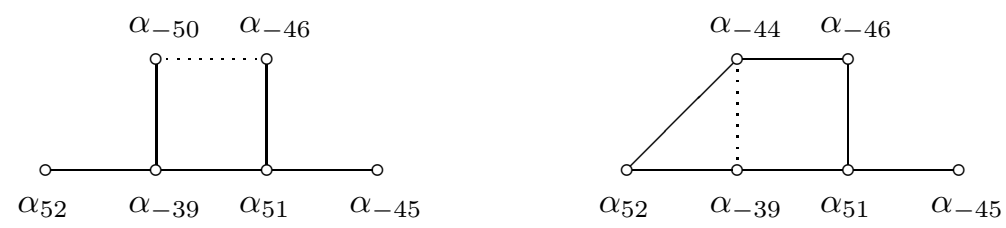

$E_{6}\left(a_{1}\right)$ : orbit 80

FIGURE 3. Diagrams for some non-standard types 

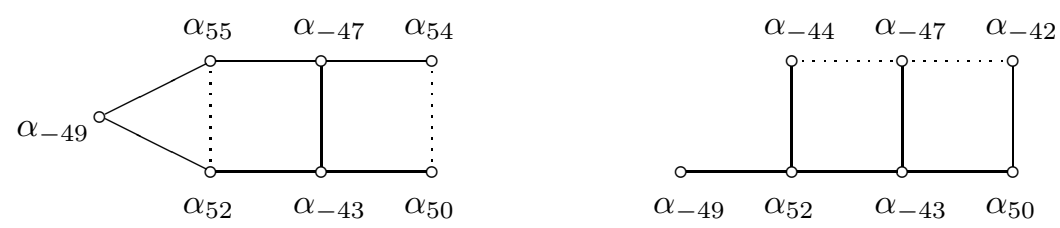

$E_{7}\left(a_{3}\right)$ : orbit 87
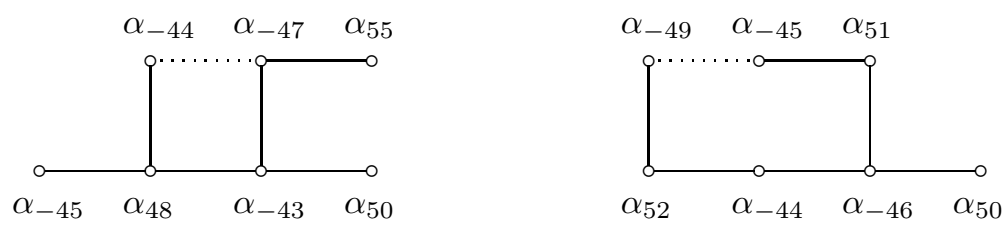

$E_{7}\left(a_{2}\right)$ : orbit 89
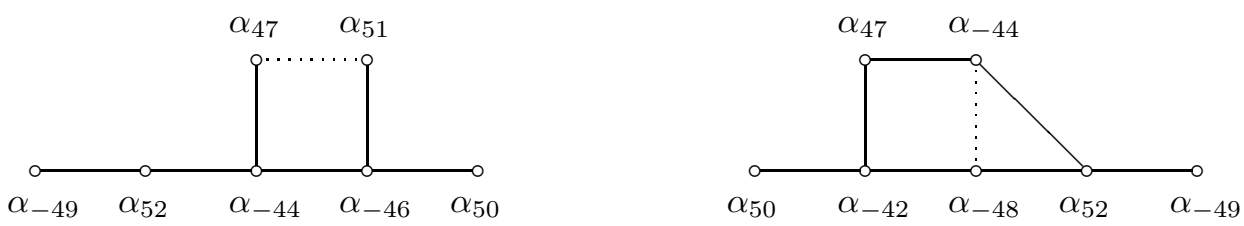

$E_{7}\left(a_{1}\right):$ orbit 91

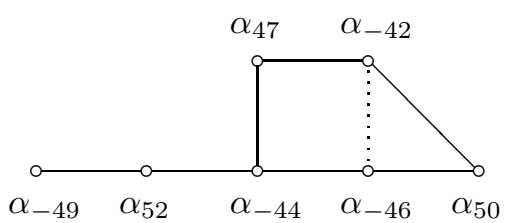

$E_{7}\left(a_{1}\right)$ : orbit 91

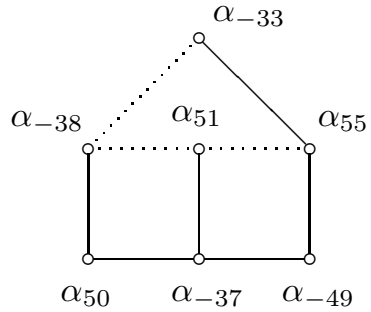

$E_{7}\left(a_{5}\right)$ : orbit 69

FIGURE 4. Diagrams for some non-standard types 
TABLE 3. Representatives of nonzero nilpotent orbits in $\mathfrak{p}\left(\mathfrak{g}_{0}=\mathrm{E} \mathrm{V}\right)$

\begin{tabular}{|c|c|c|c|c|}
\hline$k$ & $i$ & $\beta_{j}\left(H^{i}\right)$ & $E^{i} \in \mathcal{O}_{1}^{i}$ & Type \\
\hline 1 & 1 & 0001000 & $X_{-2}$ & $A_{1}$ \\
\hline 2 & 2 & 0100010 & $\left(X_{-2}\right)+\left(X_{-28}\right)$ & $2 A_{1}$ \\
\hline 3 & 3 & 0200000 & $\left(X_{-2}\right)+\left(X_{-28}\right)+\left(X_{-49}\right)$ & $\left(3 A_{1}\right)^{\prime \prime}$ \\
\hline 3 & 4 & 0000020 & $\left(X_{-2}\right)+\left(X_{-28}\right)+\left(X_{49}\right)$ & $\left(3 A_{1}\right)^{\prime \prime}$ \\
\hline 4 & 5 & 1001001 & $\left(X_{-9}\right)+\left(X_{-22}\right)+\left(X_{-33}\right)$ & $\left(3 A_{1}\right)^{\prime}$ \\
\hline 5 & 6 & 2000002 & $\begin{array}{l}X_{-2}+X_{-50} \\
\left(X_{-2}\right)+\left(X_{-28}\right)+\left(X_{-38}\right)+\left(X_{-46}\right)\end{array}$ & $\begin{array}{l}A_{2} \\
\left(4 A_{1}\right)^{\prime}\end{array}$ \\
\hline 5 & 7 & 0002000 & $\left(X_{50}\right)+\left(X_{-9}\right)+\left(X_{-22}\right)+\left(X_{-33}\right)$ & $\left(4 A_{1}\right)^{\prime}$ \\
\hline 6 & 8 & 1100100 & $\left(X_{-16}\right)+\left(X_{-20}\right)+\left(X_{-29}\right)+\left(X_{-41}\right)$ & $\left(4 A_{1}\right)^{\prime \prime}$ \\
\hline 6 & 9 & 0010011 & $\left(X_{-15}\right)+\left(X_{-23}\right)+\left(X_{-26}\right)+\left(X_{55}\right)$ & $\left(4 A_{1}\right)^{\prime \prime}$ \\
\hline 7 & 10 & 2010001 & $\begin{array}{l}\left(X_{-15}+X_{-43}\right)+\left(X_{-30}\right) \\
\left(X_{-15}\right)+\left(X_{-26}\right)+\left(X_{-30}\right)+\left(X_{-38}\right) \\
+\left(X_{-40}\right)\end{array}$ & $\begin{array}{l}A_{2}+A_{1} \\
5 A_{1}\end{array}$ \\
\hline 7 & 11 & 1000102 & $\begin{array}{l}\left(X_{-16}+X_{-42}\right)+\left(X_{59}\right) \\
\left(X_{-16}\right)+\left(X_{-29}\right)+\left(X_{59}\right)+\left(X_{-38}\right) \\
+\left(X_{-37}\right)\end{array}$ & $\begin{array}{l}A_{2}+A_{1} \\
5 A_{1}\end{array}$ \\
\hline 7 & 12 & 0101010 & $\begin{array}{l}\left(X_{57}\right)+\left(X_{-20}\right)+\left(X_{-23}\right)+\left(X_{-28}\right) \\
+\left(X_{-36}\right)\end{array}$ & $5 A_{1}$ \\
\hline 8 & 13 & 3000100 & $\begin{array}{l}\left(X_{-22}+X_{-38}\right)+\left(X_{-30}\right)+\left(X_{-47}\right) \\
\left(X_{-22}\right)+\left(X_{-30}\right)+\left(X_{-32}\right)+\left(X_{-33}\right) \\
+\left(X_{-35}\right)+\left(X_{-47}\right)\end{array}$ & $\begin{array}{l}A_{2}+2 A_{1} \\
6 A_{1}\end{array}$ \\
\hline 8 & 14 & 0010003 & $\begin{array}{l}\left(X_{-22}+X_{-38}\right)+\left(X_{59}\right)+\left(X_{47}\right) \\
\left(X_{-22}\right)+\left(X_{59}\right)+\left(X_{-35}\right)+\left(X_{-33}\right) \\
+\left(X_{-32}\right)+\left(X_{47}\right)\end{array}$ & $\begin{array}{l}A_{2}+2 A_{1} \\
6 A_{1}\end{array}$ \\
\hline 8 & 15 & 1010101 & $\begin{array}{l}\left(X_{-22}+X_{-38}\right)+\left(X_{59}\right)+\left(X_{-30}\right) \\
\left(X_{59}\right)+\left(X_{-22}\right)+\left(X_{-30}\right)+\left(X_{-32}\right) \\
+\left(X_{-33}\right)+\left(X_{-35}\right)\end{array}$ & $\begin{array}{l}A_{2}+2 A_{1} \\
6 A_{1}\end{array}$ \\
\hline 9 & 16 & 4000000 & $\begin{array}{l}\left(X_{-2}+X_{-49}\right)+\left(X_{-28}\right)+\left(X_{-38}\right)+\left(X_{-47}\right) \\
\left(X_{-2}\right)+\left(X_{-28}\right)+\left(X_{-38}\right)+\left(X_{-46}\right) \\
+\left(X_{-47}\right)+\left(X_{-48}\right)+\left(X_{-49}\right)\end{array}$ & $\begin{array}{l}A_{2}+3 A_{1} \\
7 A_{1}\end{array}$ \\
\hline 9 & 17 & 0000004 & $\begin{array}{l}\left(X_{-2}+X_{49}\right)+\left(X_{-28}\right)+\left(X_{-38}\right)+\left(X_{47}\right) \\
\left(X_{-2}\right)+\left(X_{-28}\right)+\left(X_{-38}\right)+\left(X_{-46}\right) \\
+\left(X_{47}\right)+\left(X_{48}\right)+\left(X_{49}\right)\end{array}$ & $\begin{array}{l}A_{2}+3 A_{1} \\
7 A_{1}\end{array}$ \\
\hline 9 & 18 & 2000200 & $\begin{array}{l}\left(X_{-22}+X_{-38}\right)+\left(X_{59}\right)+\left(X_{-30}\right)+\left(X_{-47}\right) \\
\left(X_{59}\right)+\left(X_{-22}\right)+\left(X_{-30}\right)+\left(X_{-32}\right) \\
+\left(X_{-33}\right)+\left(X_{-35}\right)+\left(X_{-47}\right)\end{array}$ & $\begin{array}{l}A_{2}+3 A_{1} \\
7 A_{1}\end{array}$ \\
\hline 9 & 19 & 0020002 & $\begin{array}{l}\left(X_{-22}+X_{-38}\right)+\left(X_{-30}\right)+\left(X_{59}\right)+\left(X_{47}\right) \\
\left(X_{-30}\right)+\left(X_{-22}\right)+\left(X_{59}\right)+\left(X_{-35}\right) \\
+\left(X_{-33}\right)+\left(X_{-32}\right)+\left(X_{47}\right)\end{array}$ & $\begin{array}{l}A_{2}+3 A_{1} \\
7 A_{1}\end{array}$ \\
\hline 10 & 20 & 0102010 & $X_{54}+X_{-28}+X_{-39}$ & $A_{3}$ \\
\hline 11 & 21 & 0200020 & $\left(X_{52}+X_{-23}\right)+\left(X_{-20}+X_{-45}\right)$ & $2 A_{2}$ \\
\hline 12 & 22 & 0202000 & $\left(X_{50}+X_{-28}+X_{-33}\right)+\left(X_{-49}\right)$ & $\left(A_{3}+A_{1}\right)^{\prime \prime}$ \\
\hline 12 & 23 & 0002020 & $\left(X_{50}+X_{-28}+X_{-33}\right)+\left(X_{49}\right)$ & $\left(A_{3}+A_{1}\right)^{\prime \prime}$ \\
\hline 13 & 24 & 1101011 & $\left(X_{57}+X_{-35}\right)+\left(X_{-30}+X_{-37}\right)+\left(X_{-33}\right)$ & $2 A_{2}+A_{1}$ \\
\hline 14 & 25 & 1011101 & $\left(X_{54}+X_{-28}+X_{-39}\right)+\left(X_{-38}\right)$ & $\left(A_{3}+A_{1}\right)^{\prime}$ \\
\hline
\end{tabular}


TABle 3. (continued)

\begin{tabular}{|c|c|c|c|c|}
\hline$k$ & $i$ & $\beta_{j}\left(H^{i}\right)$ & $E^{i} \in \mathcal{O}_{1}^{i}$ & Type \\
\hline 15 & 26 & 2002002 & $\left(X_{54}+X_{-28}+X_{-39}\right)+\left(X_{-38}\right)+\left(X_{-46}\right)$ & $\left(A_{3}+2 A_{1}\right)^{\prime}$ \\
\hline 15 & 27 & 0020200 & $\left(X_{46}+X_{-22}+X_{-38}\right)+\left(X_{54}\right)+\left(X_{-39}\right)$ & $\left(A_{3}+2 A_{1}\right)^{\prime}$ \\
\hline & & & $\begin{array}{l}\left(X_{46}+X_{-22}\right)+\left(X_{54}+X_{-32}\right) \\
+\left(X_{-35}+X_{-39}\right)\end{array}$ & $3 A_{2}$ \\
\hline & & & $X_{46}+X_{-22}+X_{-33}+X_{-38}$ & $D_{4}\left(a_{1}\right)$ \\
\hline 16 & 28 & 1111010 & $\left(X_{57}+X_{-41}+X_{-33}\right)+\left(X_{-32}\right)+\left(X_{-40}\right)$ & $\left(A_{3}+2 A_{1}\right)^{\prime \prime}$ \\
\hline 16 & 29 & 0101111 & $\left(X_{-36}+X_{55}+X_{-33}\right)+\left(X_{-35}\right)+\left(X_{-37}\right)$ & $\left(A_{3}+2 A_{1}\right)^{\prime \prime}$ \\
\hline 17 & 30 & 2004002 & $X_{-28}+X_{50}+X_{-38}+X_{-46}$ & $D_{4}$ \\
\hline 18 & 31 & 2101101 & $\begin{array}{l}\left(X_{59}+X_{-40}+X_{-39}\right) \\
+\left(X_{-32}\right)+\left(X_{-41}\right)+\left(X_{-42}\right)\end{array}$ & $A_{3}+3 A_{1}$ \\
\hline 18 & 32 & 1011012 & $\begin{array}{l}\left(X_{-30}+X_{-37}+X_{54}\right) \\
+\left(X_{-35}\right)+\left(X_{55}\right)+\left(X_{-43}\right)\end{array}$ & $A_{3}+3 A_{1}$ \\
\hline 18 & 33 & 0120101 & $\begin{array}{l}\left(X_{51}+X_{-36}+X_{-32}\right) \\
+\left(X_{57}\right)+\left(X_{-33}\right)+\left(X_{-40}\right)\end{array}$ & $A_{3}+3 A_{1}$ \\
\hline 18 & 34 & 1010210 & $\begin{array}{l}\left(X_{51}+X_{-26}+X_{-36}+X_{-41}\right)+\left(X_{-40}\right) \\
\left(X_{-44}+X_{57}+X_{-35}\right) \\
+\left(X_{-36}\right)+\left(X_{-33}\right)+\left(X_{-37}\right)\end{array}$ & $\begin{array}{l}D_{4}\left(a_{1}\right)+A_{1} \\
A_{3}+3 A_{1}\end{array}$ \\
\hline 19 & 35 & 1030010 & $\begin{array}{l}\left(X_{-44}+X_{-29}+X_{57}+X_{55}\right)+\left(X_{-37}\right) \\
\left(X_{-29}+X_{55}+X_{-39}\right)+\left(X_{-38}+X_{-45}\right) \\
\left(X_{55}+X_{-29}+X_{-39}+X_{-48}\right) \\
+\left(X_{-38}\right)+\left(X_{-41}\right)\end{array}$ & $\begin{array}{l}D_{4}\left(a_{1}\right)+A_{1} \\
A_{3}+A_{2} \\
D_{4}\left(a_{1}\right)+2 A_{1}\end{array}$ \\
\hline 19 & 36 & 0100301 & $\begin{array}{l}\left(X_{-26}+X_{-41}+X_{54}\right)+\left(X_{-38}+X_{52}\right) \\
\left(X_{-41}+X_{-26}+X_{54}+X_{48}\right) \\
+\left(X_{-38}\right)+\left(X_{55}\right)\end{array}$ & $\begin{array}{l}A_{3}+A_{2} \\
D_{4}\left(a_{1}\right)+2 A_{1}\end{array}$ \\
\hline 19 & 37 & 1110111 & $\begin{array}{l}\left(X_{55}+X_{-33}+X_{-36}+X_{-41}\right) \\
+\left(X_{-37}\right)+\left(X_{-40}\right)\end{array}$ & $D_{4}\left(a_{1}\right)+2 A_{1}$ \\
\hline 20 & 38 & 2200022 & $X_{-33}+X_{52}+X_{-30}+X_{-50}$ & $A_{4}$ \\
\hline 21 & 39 & 0040000 & $\begin{array}{l}\left(X_{37}+X_{-22}+X_{-38}\right)+\left(X_{59}+X_{-48}\right) \\
+\left(X_{-41}\right) \\
\left(X_{37}+X_{-22}+X_{-20}+X_{-38}\right) \\
+\left(X_{55}\right)+\left(X_{-41}\right)+\left(X_{-48}\right)\end{array}$ & $\begin{array}{l}A_{3}+A_{2}+A_{1} \\
D_{4}\left(a_{1}\right)+3 A_{1}\end{array}$ \\
\hline 21 & 40 & 0000400 & $\begin{array}{l}\left(X_{40}+X_{-22}+X_{-38}\right)+\left(X_{-30}+X_{48}\right) \\
+\left(X_{55}\right) \\
\left(X_{40}+X_{-22}+X_{-23}+X_{-38}\right) \\
+\left(X_{-41}\right)+\left(X_{55}\right)+\left(X_{48}\right)\end{array}$ & $\begin{array}{l}A_{3}+A_{2}+A_{1} \\
D_{4}\left(a_{1}\right)+3 A_{1}\end{array}$ \\
\hline 21 & 41 & 2020020 & $\begin{array}{l}\left(X_{-29}+X_{55}+X_{-39}\right)+\left(X_{-38}+X_{-45}\right) \\
+\left(X_{-37}\right) \\
\left(X_{55}+X_{-29}+X_{-39}+X_{-48}\right) \\
+\left(X_{-37}\right)+\left(X_{-38}\right)+\left(X_{-41}\right)\end{array}$ & $\begin{array}{l}A_{3}+A_{2}+A_{1} \\
D_{4}\left(a_{1}\right)+3 A_{1}\end{array}$ \\
\hline 21 & 42 & 0200202 & $\begin{array}{l}\left(X_{-26}+X_{-41}+X_{54}\right)+\left(X_{-38}+X_{52}\right) \\
+\left(X_{-40}\right) \\
\left(X_{-41}+X_{-26}+X_{54}+X_{48}\right) \\
+\left(X_{-40}\right)+\left(X_{-38}\right)+\left(X_{55}\right)\end{array}$ & $\begin{array}{l}A_{3}+A_{2}+A_{1} \\
D_{4}\left(a_{1}\right)+3 A_{1}\end{array}$ \\
\hline 20 & 43 & 0202020 & $\begin{array}{l}\left(X_{-32}+X_{50}+X_{-35}\right) \\
+\left(X_{52}+X_{-33}+X_{-45}\right)\end{array}$ & $2 A_{3}$ \\
\hline 22 & 44 & 0402020 & $X_{50}+X_{-32}+X_{-49}+X_{52}+X_{-33}$ & $\left(A_{5}\right)^{\prime \prime}$ \\
\hline
\end{tabular}


TABle 3. (continued)

\begin{tabular}{|c|c|c|c|c|}
\hline$k$ & $i$ & $\beta_{j}\left(H^{i}\right)$ & $E^{i} \in \mathcal{O}_{1}^{i}$ & Type \\
\hline 22 & 45 & 0202040 & $X_{50}+X_{-35}+X_{49}+X_{-45}+X_{-33}$ & $\left(A_{5}\right)^{\prime \prime}$ \\
\hline 23 & 46 & 2103101 & $\left(X_{-32}+X_{50}+X_{-40}+X_{-42}\right)+\left(X_{-41}\right)$ & $D_{4}+A_{1}$ \\
\hline 23 & 47 & 1013012 & $\left(X_{-35}+X_{50}+X_{-37}+X_{-43}\right)+\left(X_{55}\right)$ & $D_{4}+A_{1}$ \\
\hline 24 & 48 & 3101021 & $\left(X_{-38}+X_{57}+X_{-41}+X_{-46}\right)+\left(X_{-39}\right)$ & $A_{4}+A_{1}$ \\
\hline 24 & 49 & 1201013 & $\left(X_{-38}+X_{-36}+X_{55}+X_{-46}\right)+\left(X_{54}\right)$ & $A_{4}+A_{1}$ \\
\hline 24 & 50 & 1111111 & $\begin{array}{l}\left(X_{-37}+X_{54}+X_{-41}\right) \\
+\left(X_{55}+X_{-39}+X_{-40}\right)+\left(X_{-38}\right)\end{array}$ & $2 A_{3}+A_{1}$ \\
\hline 25 & 51 & 3013010 & $\begin{array}{l}\left(X_{-37}+X_{50}+X_{-38}+X_{-40}\right) \\
+\left(X_{-41}\right)+\left(X_{-48}\right) \\
X_{-37}+X_{50}+X_{-35}+X_{-43}+X_{-48}\end{array}$ & $\begin{array}{l}D_{4}+2 A_{1} \\
D_{5}\left(a_{1}\right)\end{array}$ \\
\hline 25 & 52 & 0103103 & $\begin{array}{l}\left(X_{-40}+X_{50}+X_{-38}+X_{-37}\right) \\
+\left(X_{55}\right)+\left(X_{48}\right) \\
X_{-40}+X_{50}+X_{-32}+X_{-42}+X_{48}\end{array}$ & $\begin{array}{l}D_{4}+2 A_{1} \\
D_{5}\left(a_{1}\right)\end{array}$ \\
\hline 25 & 53 & 1112111 & $\begin{array}{l}\left(X_{-37}+X_{50}+X_{-38}+X_{-40}\right) \\
+\left(X_{55}\right)+\left(X_{-41}\right)\end{array}$ & $D_{4}+2 A_{1}$ \\
\hline 26 & 54 & 2020202 & $\begin{array}{l}\left(X_{-37}+X_{51}+X_{-36}+X_{-43}\right) \\
+\left(X_{57}+X_{-44}\right)\end{array}$ & $A_{4}+A_{2}$ \\
\hline 27 & 55 & 4004000 & $\begin{array}{l}\left(X_{-28}+X_{50}+X_{-38}+X_{-46}\right) \\
+\left(X_{-47}\right)+\left(X_{-48}\right)+\left(X_{-49}\right) \\
\left(X_{-28}+X_{50}+X_{-38}+X_{-46}+X_{-51}\right) \\
+\left(X_{-49}\right)\end{array}$ & $\begin{array}{l}D_{4}+3 A_{1} \\
D_{5}\left(a_{1}\right)+A_{1}\end{array}$ \\
\hline 27 & 56 & 0004004 & $\begin{array}{l}\left(X_{-28}+X_{50}+X_{-38}+X_{-46}\right) \\
+\left(X_{47}\right)+\left(X_{48}\right)+\left(X_{49}\right) \\
\left(X_{-28}+X_{50}+X_{-38}+X_{-46}+X_{44}\right) \\
+\left(X_{49}\right)\end{array}$ & $\begin{array}{l}D_{4}+3 A_{1} \\
D_{5}\left(a_{1}\right)+A_{1}\end{array}$ \\
\hline 27 & 57 & 2022020 & $\begin{array}{l}\left(X_{-37}+X_{-38}+X_{50}+X_{-40}\right) \\
+\left(X_{55}\right)+\left(X_{-41}\right)+\left(X_{-48}\right) \\
\left(X_{-37}+X_{50}+X_{-35}+X_{-43}+X_{-48}\right) \\
+\left(X_{55}\right)\end{array}$ & $\begin{array}{l}D_{4}+3 A_{1} \\
D_{5}\left(a_{1}\right)+A_{1}\end{array}$ \\
\hline 27 & 58 & 0202202 & $\begin{array}{l}\left(X_{-40}+X_{-38}+X_{50}+X_{-37}\right) \\
+\left(X_{-41}\right)+\left(X_{55}\right)+\left(X_{48}\right) \\
\left(X_{-40}+X_{50}+X_{-32}+X_{-42}+X_{48}\right) \\
+\left(X_{-41}\right)\end{array}$ & $\begin{array}{l}D_{4}+3 A_{1} \\
D_{5}\left(a_{1}\right)+A_{1}\end{array}$ \\
\hline 28 & 59 & 1211121 & $X_{54}+X_{-45}+X_{-38}+X_{52}+X_{-39}$ & $\left(A_{5}\right)^{\prime}$ \\
\hline 29 & 60 & 1311111 & $\begin{array}{l}\left(X_{54}+X_{-37}+X_{-49}+X_{55}+X_{-39}\right) \\
+\left(X_{-38}\right)\end{array}$ & $\left(A_{5}+A_{1}\right)^{\prime \prime}$ \\
\hline 29 & 61 & 1111131 & $\begin{array}{l}\left(X_{-39}+X_{-40}+X_{49}+X_{-41}+X_{54}\right) \\
+\left(X_{-38}\right)\end{array}$ & $\left(A_{5}+A_{1}\right)^{\prime \prime}$ \\
\hline 30 & 62 & 2202022 & $\begin{array}{l}\left(X_{54}+X_{-45}+X_{-38}+X_{52}+X_{-39}\right) \\
+\left(X_{-46}\right)\end{array}$ & $\left(A_{5}+A_{1}\right)^{\prime}$ \\
\hline 30 & 63 & 0220220 & $\begin{array}{l}\left(X_{46}+X_{-40}+X_{-39}+X_{52}+X_{-38}\right) \\
+\left(X_{54}\right) \\
X_{54}+X_{-45}+X_{-38}+X_{52}+X_{-39}+X_{50} \\
X_{-37}+X_{46}+X_{-40}+X_{-33}+X_{52}+X_{-45}\end{array}$ & $\begin{array}{l}\left(A_{5}+A_{1}\right)^{\prime} \\
E_{6}\left(a_{3}\right) \\
E_{6}\left(a_{3}\right)\end{array}$ \\
\hline 31 & 64 & 1310301 & $\begin{array}{l}X_{-39}+X_{51}+X_{-37}+X_{-49}+X_{57}+X_{-45} \\
X_{57}+X_{-49}+X_{-37}+X_{51}+X_{-39}+X_{-44} \\
\end{array}$ & $\begin{array}{l}D_{6}\left(a_{2}\right) \\
D_{6}\left(a_{2}\right) \\
\end{array}$ \\
\hline
\end{tabular}


TABle 3. (continued)

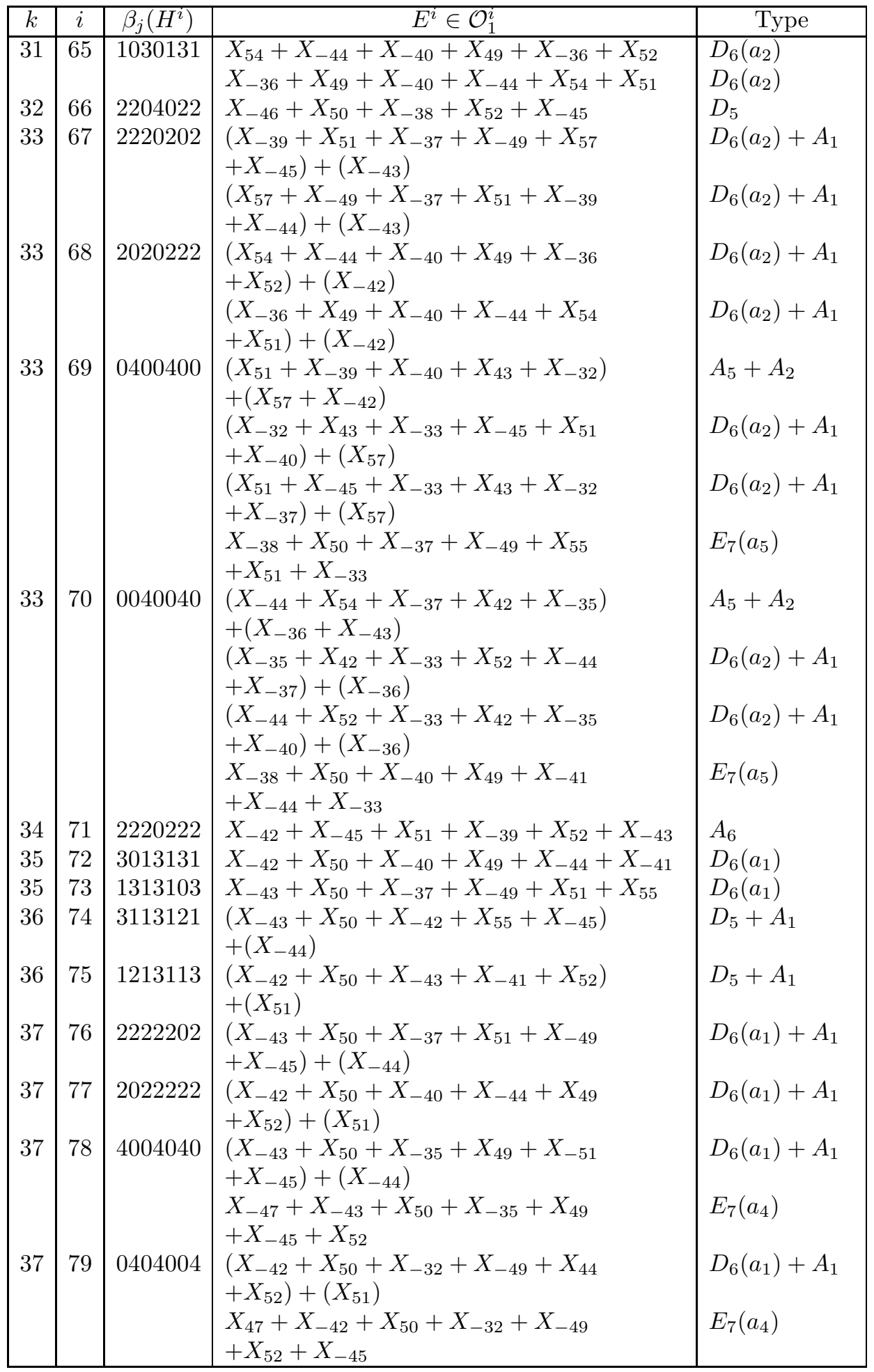


TABle 3. (continued)

\begin{tabular}{|c|c|c|c|c|}
\hline$k$ & $i$ & $\beta_{j}\left(H^{i}\right)$ & $E^{i} \in \mathcal{O}_{1}^{i}$ & Type \\
\hline 38 & 80 & 4220224 & $\begin{array}{l}X_{52}+X_{-39}+X_{51}+X_{-45}+X_{-46}+X_{-50} \\
X_{52}+X_{-39}+X_{51}+X_{-45}+X_{-46}+X_{-44}\end{array}$ & $\begin{array}{l}E_{6}\left(a_{1}\right) \\
E_{6}\left(a_{1}\right)\end{array}$ \\
\hline 38 & 81 & 2222222 & $\begin{array}{l}X_{51}+X_{-45}+X_{-42}+X_{50}+X_{-43}+X_{52} \\
+X_{-44}\end{array}$ & $A_{7}$ \\
\hline 39 & 82 & 3413131 & $X_{-42}+X_{50}+X_{-43}+X_{52}+X_{-44}+X_{-49}$ & $D_{6}$ \\
\hline 39 & 83 & 1313143 & $X_{-43}+X_{50}+X_{-42}+X_{-45}+X_{51}+X_{49}$ & $D_{6}$ \\
\hline 40 & 84 & 4224224 & $X_{52}+X_{50}+X_{-44}+X_{-46}+X_{51}+X_{-45}$ & $E_{6}$ \\
\hline 41 & 85 & 2422222 & $\begin{array}{l}\left(X_{-42}+X_{50}+X_{-43}+X_{52}+X_{-44}\right. \\
\left.+X_{-49}\right)+\left(X_{51}\right)\end{array}$ & $D_{6}+A_{1}$ \\
\hline 41 & 86 & 2222242 & $\begin{array}{l}\left(X_{-43}+X_{50}+X_{-42}+X_{-45}+X_{51}\right. \\
\left.+X_{49}\right)+\left(X_{-44}\right)\end{array}$ & $D_{6}+A_{1}$ \\
\hline 41 & 87 & 4404040 & $\begin{array}{l}\left(X_{-46}+X_{50}+X_{-38}+X_{52}+X_{-48}\right. \\
\left.+X_{-49}\right)+\left(X_{-47}\right) \\
X_{-49}+X_{52}+X_{-43}+X_{50}+X_{55} \\
+X_{-47}+X_{54} \\
X_{-46}+X_{50}+X_{-38}+X_{52}+X_{-49} \\
+X_{-38}+X_{-51}\end{array}$ & $\begin{array}{l}D_{6}+A_{1} \\
E_{7}\left(a_{3}\right) \\
E_{7}\left(a_{3}\right)\end{array}$ \\
\hline 41 & 88 & 0404044 & $\begin{array}{l}\left(X_{-46}+X_{50}+X_{-38}+X_{-45}+X_{48}\right. \\
\left.+X_{49}\right)+\left(X_{47}\right) \\
X_{49}+X_{-45}+X_{-42}+X_{50}+X_{-41} \\
+X_{47}+X_{-39} \\
X_{-46}+X_{50}+X_{-38}+X_{-45}+X_{49} \\
+X_{-38}+X_{44}\end{array}$ & $\begin{array}{l}D_{6}+A_{1} \\
E_{7}\left(a_{3}\right) \\
E_{7}\left(a_{3}\right)\end{array}$ \\
\hline 42 & 89 & 4404404 & $\begin{array}{l}X_{-45}+X_{50}+X_{48}+X_{-43}+X_{-47}+X_{55} \\
+X_{-44} \\
X_{52}+X_{50}+X_{-44}+X_{-46}+X_{51}+X_{-45} \\
+X_{-49}\end{array}$ & $\begin{array}{l}E_{7}\left(a_{2}\right) \\
E_{7}\left(a_{2}\right)\end{array}$ \\
\hline 42 & 90 & 4044044 & $\begin{array}{l}X_{52}+X_{50}+X_{-48}+X_{-42}+X_{47}+X_{-41} \\
+X_{51} \\
X_{-45}+X_{50}+X_{51}+X_{-46}+X_{-44}+X_{52} \\
+X_{49}\end{array}$ & $\begin{array}{l}E_{7}\left(a_{2}\right) \\
E_{7}\left(a_{2}\right)\end{array}$ \\
\hline 43 & 91 & 4444044 & $\begin{array}{l}X_{-49}+X_{47}+X_{52}+X_{-44}+X_{-46}+X_{50} \\
+X_{51} \\
X_{50}+X_{-42}+X_{47}+X_{-44}+X_{52}+X_{-49} \\
+X_{-48} \\
X_{50}+X_{-42}+X_{47}+X_{-44}+X_{52}+X_{-49} \\
+X_{-46}\end{array}$ & $\begin{array}{l}E_{7}\left(a_{1}\right) \\
E_{7}\left(a_{1}\right) \\
E_{7}\left(a_{1}\right)\end{array}$ \\
\hline 43 & 92 & 4404444 & $\begin{array}{l}X_{49}+X_{-47}+X_{-45}+X_{51}+X_{-46}+X_{50} \\
+X_{-44} \\
X_{50}+X_{-43}+X_{-47}+X_{51}+X_{-45}+X_{49} \\
+X_{48} \\
X_{50}+X_{-43}+X_{-47}+X_{51}+X_{-45}+X_{49} \\
+X_{-46}\end{array}$ & $\begin{array}{l}E_{7}\left(a_{1}\right) \\
E_{7}\left(a_{1}\right) \\
E_{7}\left(a_{1}\right)\end{array}$ \\
\hline 44 & 93 & 8444444 & $\begin{array}{l}X_{50}+X_{-47}+X_{-46}+X_{51}+X_{-48}+X_{52} \\
+X_{-49}\end{array}$ & $E_{7}$ \\
\hline
\end{tabular}


TABLE 3. (continued)

\begin{tabular}{|c|c|c|c|c|}
\hline$k$ & $i$ & $\beta_{j}\left(H^{i}\right)$ & \multicolumn{1}{|c|}{$E^{i} \in \mathcal{O}_{1}^{i}$} & Type \\
\hline 44 & 94 & 4444448 & $\begin{array}{l}X_{50}+X_{47}+X_{-46}+X_{-44}+X_{48}+X_{-45} \\
+X_{49}\end{array}$ & $E_{7}$ \\
\hline
\end{tabular}

\section{Description of the ClOSURE Diagram}

We can now state our main result.

Theorem 4.1. Let $\mathfrak{g}_{0}$ be of type EV. Then the closure ordering of the nilpotent $K^{0}$-orbits in $\mathfrak{p}$ is as given in Figures 5 and 6 .

For typographical reasons the closure diagram is split into two parts. The top part is shown in Figure 5 and the bottom part in Figure 6. From now on the word diagram will refer (unless stated otherwise) to the diagram exhibited in these two figures. The orbit $\mathcal{O}_{1}^{i}$ is represented by the node with label $i$.

The numbers on the left-hand side of the diagram are the complex dimensions of the orbits on that level. On the right-hand side we indicate the action of $\tilde{w}$ on $\mathcal{N}_{1} / K^{0}$. We write $i \smile j$ to indicate that $\tilde{w}\left(\mathcal{O}_{1}^{i}\right)=\mathcal{O}_{1}^{j}$ (see also Table 2 ). The part of the diagram that is fixed by $\tilde{w}$ is highlighted by using thicker bonds and nodes.

\section{JUSTIFICATION FOR THE LINES IN THE DIAGRAM}

We write $\mathcal{O}_{1}^{i} \geq \mathcal{O}_{1}^{j}$ if $\overline{\mathcal{O}_{1}^{i}} \supset \mathcal{O}_{1}^{j}$, and $\mathcal{O}_{1}^{i}>\mathcal{O}_{1}^{j}$ if $\mathcal{O}_{1}^{i} \neq \mathcal{O}_{1}^{j}$. In this section we prove that if $i$ and $j$ are two nodes in the diagram, with $i$ above $j$, which are joined by a line, then $\mathcal{O}_{1}^{i}>\mathcal{O}_{1}^{j}$. Clearly, we may assume that $j \neq 0$. If $\mathcal{O}_{1}^{i}>\mathcal{O}_{1}^{j}$, then $\tilde{w}\left(\mathcal{O}_{1}^{i}\right)>\tilde{w}\left(\mathcal{O}_{1}^{j}\right)$. This reduces considerably the number of cases that we need to consider.

Since $\mathfrak{p}_{2}\left(H^{i}\right) \subset \overline{\mathcal{O}_{1}^{i}}$, in order to prove that $\mathcal{O}_{1}^{i}>\mathcal{O}_{1}^{j}$ it suffices to exhibit an element $E \in \mathfrak{p}_{2}\left(H^{i}\right) \cap \mathcal{O}_{1}^{j}$. Such elements are given in Table 4. The fact that $E \in \mathfrak{p}_{2}\left(H^{i}\right)$ is easy to verify by using Table 7 (see the Appendix).

It is more complicated to verify that $E \in \mathcal{O}_{1}^{j}$. By using Table 7, one can easily determine whether or not $E$ belongs to $\mathfrak{g}_{H^{j}}(1,2)$. If this is the case, then $E \in \mathcal{O}_{1}^{j}$ holds if and only if $\operatorname{dim}\left(K^{0} \cdot E\right)=\operatorname{dim}\left(\mathcal{O}_{1}^{j}\right)$. The last condition is easy to test because the dimension of $K^{0} \cdot E$ is determined by the type of element $E$ given in the last column of the table. (Alternatively, one can just compute the dimension of $K^{0} \cdot E$.) This method is applicable to all entries of Table 4 except when $(i, j)$ is one of the following pairs:

$$
\begin{array}{lllll}
(60,54), & (59,54), & (64,59), & (87,80), & (44,38), \\
(83,78), & (94,91), & (90,85), & (51,48), & (43,35) .
\end{array}
$$

In the cases $(60,54)$ and $(59,54) E$ is of type $A_{4}+A_{2}$ and so $E \in \mathcal{O}^{26}$. Since $\mathcal{O}^{26} \cap \mathfrak{p}=\mathcal{O}_{1}^{54}$, we have $E \in \mathcal{O}_{1}^{54}$. The same argument is valid for the pair $(64,59)$ where $E$ is of type $\left(A_{5}\right)^{\prime}$.

In the case $(87,80) E$ is of type $E_{6}\left(a_{1}\right)$ and so $E \in \mathcal{O}^{38}$. As $\mathcal{O}^{38} \cap \mathfrak{p}=\mathcal{O}_{1}^{80} \cup \mathcal{O}_{1}^{81}$ and only the orbit $\mathcal{O}_{1}^{80}$ has a representative of type $E_{6}\left(a_{1}\right)$, we conclude that $E \in$ $\mathcal{O}_{1}^{80}$. This argument is also applicable to the pair $(44,38)$.

Let us consider in detail the pair $(83,78)$. In this case

$$
E=X_{-42}+X_{-45}+X_{51}+X_{-39}+X_{52}+X_{-43}+X_{49} .
$$

In the table, its type is given as $E_{7}\left(a_{4}\right)$. 


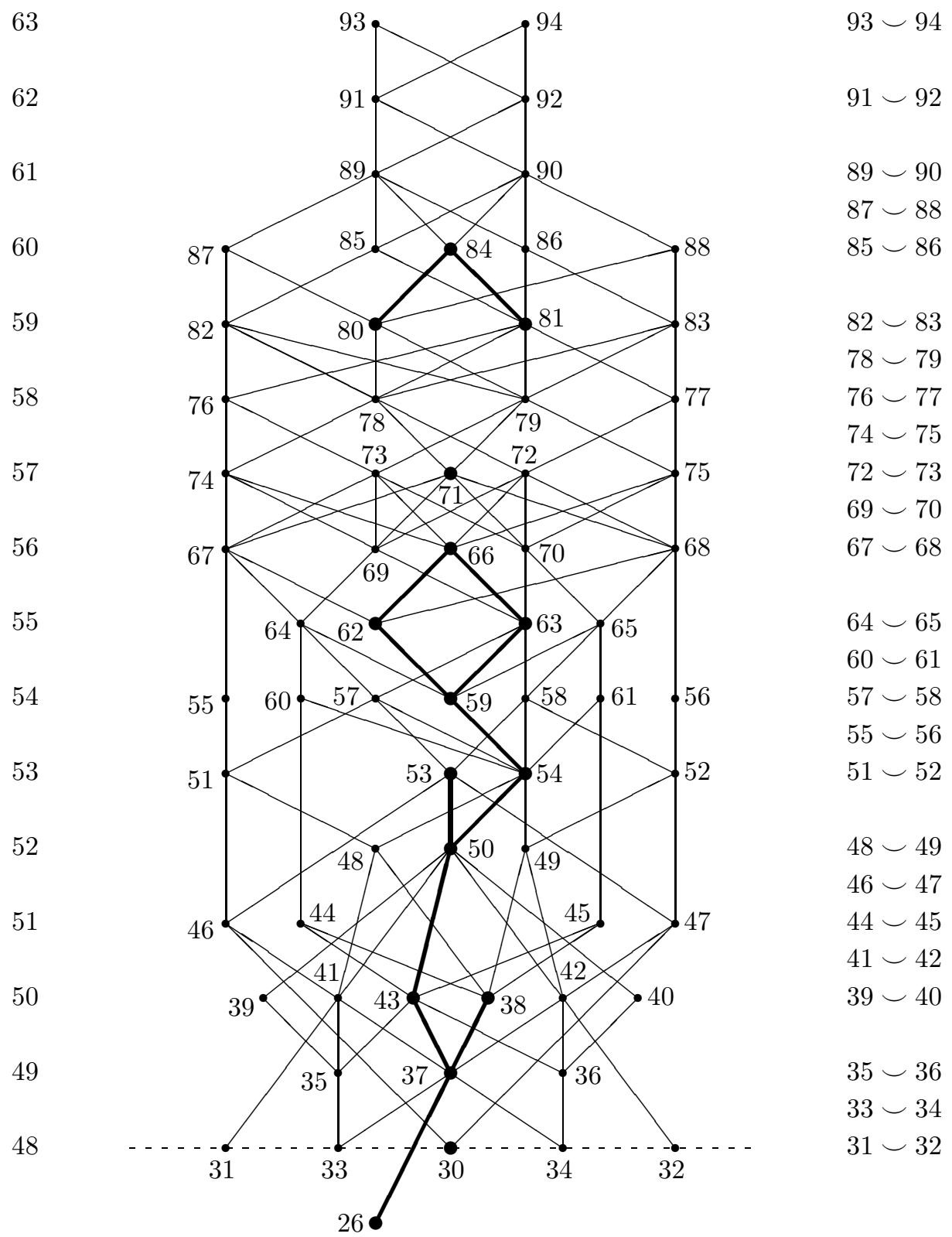

Figure 5. The closure diagram of E V (top) 


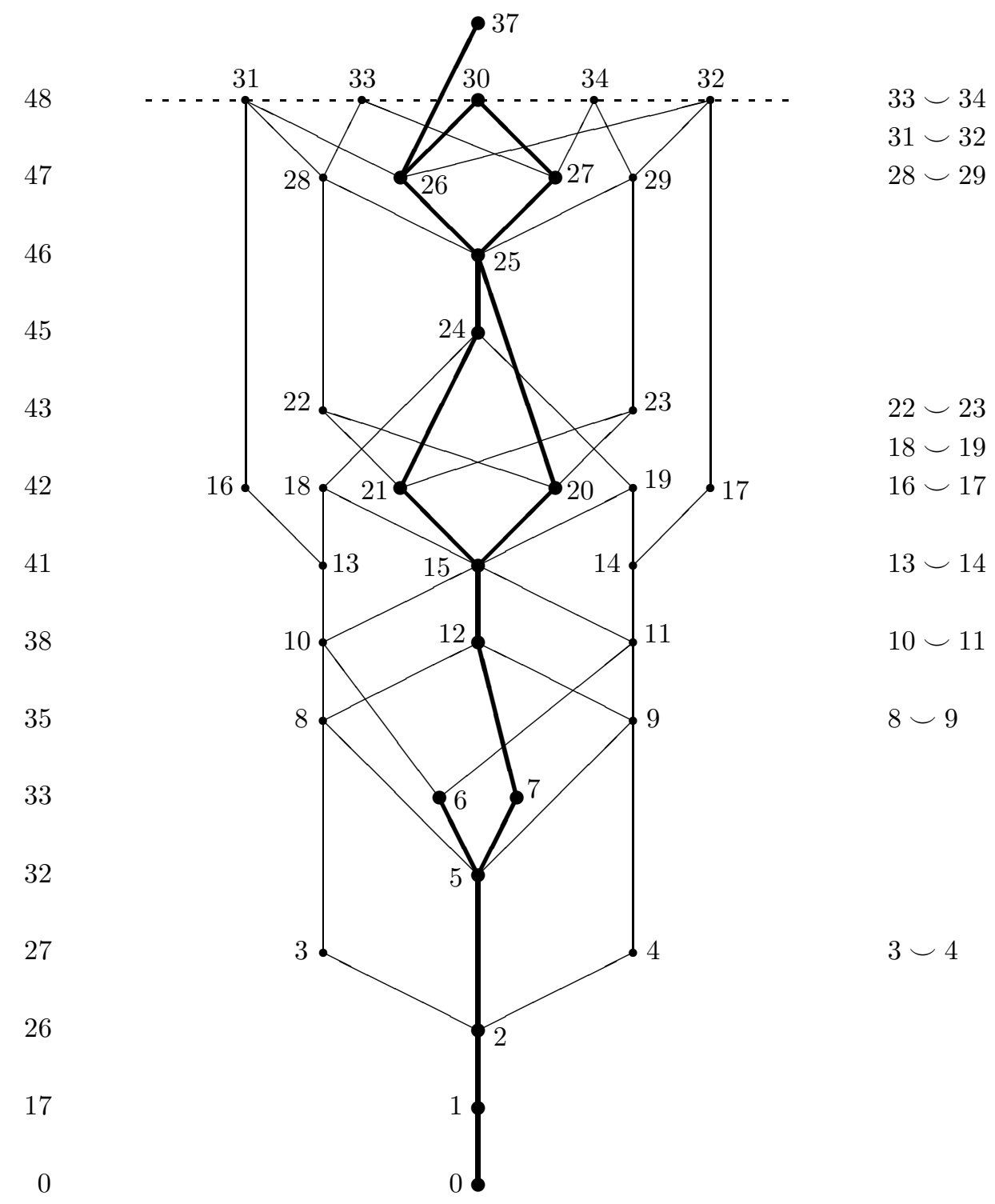

FiguRE 6 . The closure diagram of E V (bottom) 
The Dynkin-like diagram for the set of roots

$$
\Delta=\left\{\alpha_{-42}, \alpha_{-45}, \alpha_{51}, \alpha_{-39}, \alpha_{52}, \alpha_{-43}, \alpha_{49}\right\}
$$

is shown in Figure 7. The dotted line indicates that the angle between the roots $\alpha_{49}$ and $\alpha_{52}$ is $\pi / 3$.

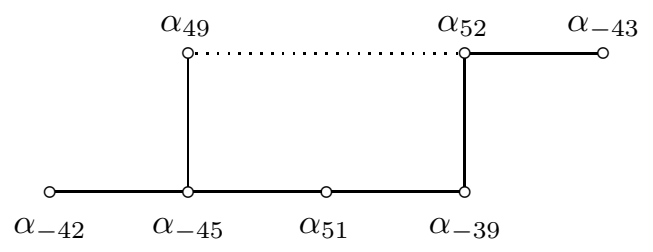

Figure 7. Type $E_{7}\left(a_{4}\right)$

A computation shows that $\operatorname{dim}\left(K^{0} \cdot E\right)=58$, and so $E$ indeed belongs to the orbit $\mathcal{O}^{37}$ with Bala-Carter symbol $E_{7}\left(a_{4}\right)$. The intersection of this orbit with $\mathfrak{p}$ is the union of four $K^{0}$-orbits: $\mathcal{O}_{1}^{76}, \mathcal{O}_{1}^{77}, \mathcal{O}_{1}^{78}$, and $\mathcal{O}_{1}^{79}$. Let $H \in \mathfrak{h}$ be the unique element such that

$$
\alpha_{m}(H)=0,-10,4,-4,4,4,0 \quad(1 \leq m \leq 7) .
$$

It follows that

$$
\beta_{m}(H)=4,0,4,-4,4,4,0 \quad(1 \leq m \leq 7) .
$$

Then $\alpha(H)=2$ for all $\alpha \in \Delta$. By applying a suitable element of the Weyl group of $(\mathfrak{k}, \mathfrak{h})$ to $H$, we obtain the element $H^{\prime} \in \mathfrak{h}$ such that

$$
\beta_{m}\left(H^{\prime}\right)=4,0,0,4,0,4,0 \quad(1 \leq m \leq 7) .
$$

Hence $H^{\prime}=H^{78}$.

Since $\alpha(H)=2$ for all $\alpha \in \Delta$, we have $E \in \mathfrak{g}_{H}(1,2)$. Since $H^{\prime}=H^{78}$, this implies that $E$ belongs to the closure of $\mathcal{O}_{1}^{78}$. As $\operatorname{dim}\left(K^{0} \cdot E\right)=58=\operatorname{dim}\left(\mathcal{O}_{1}^{78}\right)$, we infer that $E \in \mathcal{O}_{1}^{78}$.

For the remaining four pairs $(i, j)$ the argument is similar. We just give the element $H \in \mathfrak{h}$ used in each case:

$$
\begin{array}{llll}
(94,91): & \alpha_{m}(H)=4,-26,4,4,4,-4,8 \quad(1 \leq m \leq 7), \\
(90,85): & \alpha_{m}(H)=6,-16,4,4,-6,4,4 \quad(1 \leq m \leq 7), \\
(51,48): & \alpha_{m}(H)=1,-7,0,1,1,1,-3 \quad(1 \leq m \leq 7), \\
(43,35): & \alpha_{m}(H)=0,-6,3,0,1,0,-1 \quad(1 \leq m \leq 7) .
\end{array}
$$

An alternative and simpler argument can be used for the pair $(94,91)$ : The reflection corresponding to the simple root $\beta_{6}$ of $R_{0}$ transforms the diagram of the element $E$ from Table 4 into the diagram of the representative $E^{91}$ from Table 3. 
TABLE 4. Elements $E \in \mathfrak{p}_{2}\left(H^{i}\right) \cap \mathcal{O}_{1}^{j}$

\begin{tabular}{|c|c|c|c|}
\hline$i$ & $j$ & $E$ & Type \\
\hline 94 & 91 & $X_{-45}+X_{47}+X_{48}+X_{-44}+X_{-46}+X_{50}+X_{55}$ & $E_{7}\left(a_{1}\right)$ \\
\hline 93 & 91 & $\begin{array}{l}X_{-49}+X_{51}+X_{52}+X_{-48}+X_{-42}+X_{50} \\
+X_{-46}\end{array}$ & $E_{7}\left(a_{1}\right)$ \\
\hline 92 & 89 & $\begin{array}{l}X_{55}+X_{50}+X_{-47}+X_{-43}+X_{48}+X_{-45} \\
+X_{-44}\end{array}$ & $E_{7}\left(a_{2}\right)$ \\
\hline 91 & 89 & $\begin{array}{l}X_{52}+X_{50}+X_{-44}+X_{-46}+X_{51}+X_{-45} \\
+X_{-49}\end{array}$ & $E_{7}\left(a_{2}\right)$ \\
\hline 93 & 87 & $\begin{array}{l}\left(X_{-46}+X_{50}+X_{-38}+X_{52}+X_{-48}+X_{-49}\right) \\
+\left(X_{-47}\right)\end{array}$ & $D_{6}+A_{1}$ \\
\hline 89 & 87 & $\begin{array}{l}X_{-42}+X_{50}+X_{-43}+X_{52}+X_{-49}+X_{-44} \\
+X_{-47}\end{array}$ & $E_{7}\left(a_{3}\right)$ \\
\hline 90 & 85 & $\begin{array}{l}\left(X_{52}+X_{-48}+X_{51}+X_{-46}+X_{50}+X_{-41}\right) \\
+\left(X_{-20}\right)\end{array}$ & $D_{6}+A_{1}$ \\
\hline 89 & 85 & $\begin{array}{l}\left(X_{-42}+X_{50}+X_{-43}+X_{52}+X_{-44}+X_{-49}\right) \\
+\left(X_{51}\right)\end{array}$ & $D_{6}+A_{1}$ \\
\hline 89 & 84 & $X_{52}+X_{50}+X_{-44}+X_{-46}+X_{51}+X_{-45}$ & $E_{6}$ \\
\hline 85,87 & 82 & $X_{-42}+X_{50}+X_{-43}+X_{52}+X_{-44}+X_{-49}$ & $D_{6}$ \\
\hline 84,85 & 81 & $\begin{array}{l}X_{51}+X_{-45}+X_{-42}+X_{50}+X_{-43}+X_{52} \\
+X_{-44}\end{array}$ & $A_{7}$ \\
\hline 87 & 80 & $X_{-49}+X_{52}+X_{-43}+X_{50}+X_{-47}+X_{55}$ & $E_{6}\left(a_{1}\right)$ \\
\hline 84 & 80 & $X_{52}+X_{-39}+X_{51}+X_{-45}+X_{-46}+X_{-44}$ & $E_{6}\left(a_{1}\right)$ \\
\hline 83 & 78 & $\begin{array}{l}X_{-42}+X_{-45}+X_{51}+X_{-39}+X_{52}+X_{-43} \\
+X_{49}\end{array}$ & $E_{7}\left(a_{4}\right)$ \\
\hline 82,81 & 78 & $\begin{array}{l}X_{-44}+X_{52}+X_{-43}+X_{50}+X_{-42}+X_{-45} \\
+X_{55}\end{array}$ & $E_{7}\left(a_{4}\right)$ \\
\hline 80 & 78 & $\begin{array}{l}\left(X_{-45}+X_{54}+X_{-44}+X_{52}+X_{-46}+X_{-43}\right) \\
+\left(X_{-35}\right)\end{array}$ & $D_{6}\left(a_{1}\right)+A_{1}$ \\
\hline 82 & 76 & $\begin{array}{l}\left(X_{-43}+X_{50}+X_{-42}+X_{55}+X_{-45}+X_{-49}\right) \\
+\left(X_{-44}\right)\end{array}$ & $D_{6}\left(a_{1}\right)+A_{1}$ \\
\hline 81 & 76 & $\begin{array}{l}\left(X_{-43}+X_{50}+X_{-42}+X_{-45}+X_{55}+X_{51}\right) \\
+\left(X_{-44}\right)\end{array}$ & $D_{6}\left(a_{1}\right)+A_{1}$ \\
\hline 76,78 & 74 & $\left(X_{-43}+X_{50}+X_{-42}+X_{55}+X_{-45}\right)+\left(X_{-44}\right)$ & $D_{5}+A_{1}$ \\
\hline 76,79 & 73 & $X_{-43}+X_{50}+X_{-37}+X_{51}+X_{-49}+X_{-45}$ & $D_{6}\left(a_{1}\right)$ \\
\hline 78,81 & 71 & $X_{-42}+X_{-45}+X_{54}+X_{-44}+X_{52}+X_{-43}$ & $A_{6}$ \\
\hline 74 & 69 & $\begin{array}{l}\left(X_{55}+X_{-42}+X_{-45}+X_{54}+X_{-44}+X_{50}\right) \\
+\left(X_{-26}\right)\end{array}$ & $D_{6}\left(a_{2}\right)+A_{1}$ \\
\hline 73 & 69 & $\begin{array}{l}X_{55}+X_{-49}+X_{-37}+X_{50}+X_{-38}+X_{51} \\
+X_{-33}\end{array}$ & $E_{7}\left(a_{5}\right)$ \\
\hline 72 & 69 & $\begin{array}{l}\left(X_{52}+X_{-44}+X_{-40}+X_{50}+X_{-42}+X_{54}\right) \\
+\left(X_{-26}\right)\end{array}$ & $D_{6}\left(a_{2}\right)+A_{1}$ \\
\hline 71 & 69 & $\begin{array}{l}\left(X_{-44}+X_{54}+X_{-45}+X_{-42}+X_{55}+X_{52}\right) \\
+\left(X_{-26}\right)\end{array}$ & $D_{6}\left(a_{2}\right)+A_{1}$ \\
\hline 71,74 & 67 & $\begin{array}{l}\left(X_{-44}+X_{54}+X_{-45}+X_{-42}+X_{55}+X_{-37}\right) \\
+\left(X_{-43}\right)\end{array}$ & $D_{6}\left(a_{2}\right)+A_{1}$ \\
\hline
\end{tabular}


TABle 4. (continued)

\begin{tabular}{|c|c|c|c|}
\hline$i$ & $j$ & $E$ & Type \\
\hline 73 & 67 & $\begin{array}{l}\left(X_{-39}+X_{55}+X_{-42}+X_{-45}+X_{54}+X_{-49}\right) \\
+\left(X_{-43}\right)\end{array}$ & $D_{6}\left(a_{2}\right)+A_{1}$ \\
\hline 73,74 & 66 & $X_{-43}+X_{50}+X_{-42}+X_{55}+X_{-45}$ & $D_{5}$ \\
\hline 67,69 & 64 & $X_{-39}+X_{51}+X_{-37}+X_{-49}+X_{57}+X_{-45}$ & $D_{6}\left(a_{2}\right)$ \\
\hline 69 & 63 & $\left(X_{46}+X_{-40}+X_{-39}+X_{52}+X_{-38}\right)+\left(X_{54}\right)$ & $\left(A_{5}+A_{1}\right)^{\prime}$ \\
\hline 66 & 63 & $X_{54}+X_{-45}+X_{-38}+X_{52}+X_{-39}+X_{50}$ & $E_{6}\left(a_{3}\right)$ \\
\hline 66,67 & 62 & $\left(X_{54}+X_{-45}+X_{-42}+X_{55}+X_{-39}\right)+\left(X_{-43}\right)$ & $\left(A_{5}+A_{1}\right)^{\prime}$ \\
\hline 64 & 60 & $\left(X_{54}+X_{-37}+X_{-49}+X_{55}+X_{-39}\right)+\left(X_{-38}\right)$ & $\left(A_{5}+A_{1}\right)^{\prime \prime}$ \\
\hline 64 & 59 & $X_{57}+X_{-49}+X_{-37}+X_{51}+X_{-39}$ & $\left(A_{5}\right)^{\prime}$ \\
\hline 62,63 & 59 & $X_{54}+X_{-45}+X_{-38}+X_{52}+X_{-39}$ & $\left(A_{5}\right)^{\prime}$ \\
\hline 63,64 & 57 & $\left(X_{-37}+X_{54}+X_{-45}+X_{-44}+X_{-38}\right)+\left(X_{55}\right)$ & $D_{5}\left(a_{1}\right)+A_{1}$ \\
\hline 67 & 55 & $\left(X_{-44}+X_{54}+X_{-37}+X_{-45}+X_{-49}\right)+\left(X_{-43}\right)$ & $D_{5}\left(a_{1}\right)+A_{1}$ \\
\hline 59,60 & 54 & $\left(X_{-37}+X_{54}+X_{-45}+X_{-38}\right)+\left(X_{55}+X_{-39}\right)$ & $A_{4}+A_{2}$ \\
\hline 57 & 54 & $\left(X_{-37}+X_{54}+X_{-44}+X_{-40}\right)+\left(X_{55}+X_{-36}\right)$ & $A_{4}+A_{2}$ \\
\hline 57 & 53 & $\left(X_{-37}+X_{50}+X_{-38}+X_{-40}\right)+\left(X_{55}\right)+\left(X_{-41}\right)$ & $D_{4}+2 A_{1}$ \\
\hline 55,57 & 51 & $\begin{array}{l}\left(X_{-35}+X_{50}+X_{-37}+X_{-43}\right)+\left(X_{-44}\right) \\
+\left(X_{-45}\right)\end{array}$ & $D_{4}+2 A_{1}$ \\
\hline 53,54 & 50 & $\begin{array}{l}\left(X_{55}+X_{-39}+X_{-40}\right)+\left(X_{-37}+X_{54}+X_{-41}\right) \\
+\left(X_{-38}\right)\end{array}$ & $2 A_{3}+A_{1}$ \\
\hline 54 & 48 & $\left(X_{-42}+X_{57}+X_{-41}+X_{-43}\right)+\left(X_{-39}\right)$ & $A_{4}+A_{1}$ \\
\hline 51 & 48 & $\left(X_{-37}+X_{50}+X_{-35}+X_{-48}\right)+\left(X_{-33}\right)$ & $A_{4}+A_{1}$ \\
\hline 51,53 & 46 & $\left(X_{-37}+X_{50}+X_{-38}+X_{-40}\right)+\left(X_{-41}\right)$ & $D_{4}+A_{1}$ \\
\hline 60 & 44 & $X_{54}+X_{-37}+X_{-49}+X_{55}+X_{-39}$ & $\left(A_{5}\right)^{\prime \prime}$ \\
\hline 44,50 & 43 & $\left(X_{55}+X_{-39}+X_{-40}\right)+\left(X_{-37}+X_{54}+X_{-41}\right)$ & $2 A_{3}$ \\
\hline $46,48,50$ & 41 & $\begin{array}{l}\left(X_{-38}+X_{57}+X_{-41}\right)+\left(X_{-39}+X_{-40}\right) \\
+\left(X_{-37}\right)\end{array}$ & $A_{3}+A_{2}+A_{1}$ \\
\hline 50 & 39 & $\left(X_{55}+X_{-39}+X_{-40}\right)+\left(X_{54}+X_{-41}\right)+\left(X_{-38}\right)$ & $A_{3}+A_{2}+A_{1}$ \\
\hline 48 & 38 & $X_{-38}+X_{57}+X_{-41}+X_{-46}$ & $A_{4}$ \\
\hline 44 & 38 & $X_{-32}+X_{-49}+X_{52}+X_{-33}$ & $A_{4}$ \\
\hline $38,41,43$ & 37 & $\begin{array}{l}\left(X_{55}+X_{-33}+X_{-36}+X_{-41}\right)+\left(X_{-37}\right) \\
+\left(X_{-40}\right)\end{array}$ & $D_{4}\left(a_{1}\right)+2 A_{1}$ \\
\hline 43 & 35 & $\left(X_{-32}+X_{50}+X_{-35}\right)+\left(X_{-33}+X_{-45}\right)$ & $A_{3}+A_{2}$ \\
\hline 39,41 & 35 & $\left(X_{-29}+X_{55}+X_{-39}\right)+\left(X_{-38}+X_{-45}\right)$ & $A_{3}+A_{2}$ \\
\hline 35,37 & 33 & $\left(X_{55}+X_{-33}+X_{-36}+X_{-41}\right)+\left(X_{-40}\right)$ & $D_{4}\left(a_{1}\right)+A_{1}$ \\
\hline 41 & 31 & $\begin{array}{l}\left(X_{59}+X_{-40}+X_{-39}\right)+\left(X_{-37}\right)+\left(X_{-38}\right) \\
+\left(X_{-41}\right)\end{array}$ & $A_{3}+3 A_{1}$ \\
\hline 46 & 30 & $X_{-32}+X_{50}+X_{-40}+X_{-42}$ & $D_{4}$ \\
\hline 33 & 28 & $\left(X_{57}+X_{-41}+X_{-33}\right)+\left(X_{-32}\right)+\left(X_{-40}\right)$ & $\left(A_{3}+2 A_{1}\right)^{\prime \prime}$ \\
\hline 31 & 28 & $\left(X_{59}+X_{-40}+X_{-39}\right)+\left(X_{-32}\right)+\left(X_{-41}\right)$ & $\left(A_{3}+2 A_{1}\right)^{\prime \prime}$ \\
\hline 33 & 27 & $\left(X_{51}+X_{-36}+X_{-32}\right)+\left(X_{57}\right)+\left(X_{-33}\right)$ & $\left(A_{3}+2 A_{1}\right)^{\prime}$ \\
\hline 30 & 27 & $\left(X_{-32}+X_{50}+X_{-35}\right)+\left(X_{-22}\right)+\left(X_{-33}\right)$ & $\left(A_{3}+2 A_{1}\right)^{\prime}$ \\
\hline 37 & 26 & $\left(X_{57}+X_{-38}+X_{-36}\right)+\left(X_{-37}\right)+\left(X_{-40}\right)$ & $\left(A_{3}+2 A_{1}\right)^{\prime}$ \\
\hline 30,31 & 26 & $\left(X_{59}+X_{-40}+X_{-39}\right)+\left(X_{-32}\right)+\left(X_{-42}\right)$ & $\left(A_{3}+2 A_{1}\right)^{\prime}$ \\
\hline $26,27,28$ & 25 & $\left(X_{57}+X_{-35}+X_{-39}\right)+\left(X_{-32}\right)$ & $\left(A_{3}+A_{1}\right)^{\prime}$ \\
\hline 25 & 24 & $\left(X_{57}+X_{-35}\right)+\left(X_{-32}+X_{-36}\right)+\left(X_{-33}\right)$ & $2 A_{2}+A_{1}$ \\
\hline 28 & 22 & $\left(X_{57}+X_{-41}+X_{-33}\right)+\left(X_{-40}\right)$ & $\left(A_{3}+A_{1}\right)^{\prime \prime}$ \\
\hline
\end{tabular}


TABLE 4. (continued)

\begin{tabular}{|r|c|l|l|}
\hline \multicolumn{1}{|c|}{$i$} & $j$ & \multicolumn{1}{|c|}{$E$} & \multicolumn{1}{c|}{ Type } \\
\hline 24 & 21 & $\left(X_{57}+X_{-35}\right)+\left(X_{-30}+X_{-37}\right)$ & $2 A_{2}$ \\
22 & 21 & $\left(X_{57}+X_{-35}\right)+\left(X_{-20}+X_{-45}\right)$ & $2 A_{2}$ \\
22,25 & 20 & $X_{54}+X_{-28}+X_{-39}$ & $A_{3}$ \\
24 & 18 & $\left(X_{-30}+X_{-37}\right)+\left(X_{59}\right)+\left(X_{-33}\right)+\left(X_{-35}\right)$ & $A_{2}+3 A_{1}$ \\
31 & 16 & $\left(X_{-39}+X_{-40}\right)+\left(X_{-32}\right)+\left(X_{-41}\right)+\left(X_{-42}\right)$ & $A_{2}+3 A_{1}$ \\
18,21 & 15 & $\left(X_{-26}+X_{-35}\right)+\left(X_{59}\right)+\left(X_{-30}\right)$ & $A_{2}+2 A_{1}$ \\
20 & 15 & $\left(X_{-28}+X_{-33}\right)+\left(X_{59}\right)+\left(X_{-30}\right)$ & $A_{2}+2 A_{1}$ \\
16,18 & 13 & $\left(X_{-29}+X_{-44}\right)+\left(X_{-16}\right)+\left(X_{-37}\right)$ & $A_{2}+2 A_{1}$ \\
15 & 12 & $\left(X_{59}+\left(X_{-26}\right)+\left(X_{-28}\right)+\left(X_{-29}\right)+\left(X_{-30}\right)\right.$ & $5 A_{1}$ \\
15 & 10 & $\left(X_{-26}\right)+\left(X_{-28}\right)+\left(X_{-29}\right)+\left(X_{-30}\right)+\left(X_{-38}\right)$ & $5 A_{1}$ \\
13 & 10 & $\left(X_{-22}+X_{-38}\right)+\left(X_{-30}\right)$ & $A_{2}+A_{1}$ \\
10,12 & 8 & $\left(X_{-20}\right)+\left(X_{-28}\right)+\left(X_{-29}\right)+\left(X_{-30}\right)$ & $\left(4 A_{1}\right)^{\prime \prime}$ \\
12 & 7 & $\left(X_{59}\right)+\left(X_{-26}\right)+\left(X_{-29}\right)+\left(X_{-30}\right)$ & $\left(4 A_{1}\right)^{\prime}$ \\
10 & 6 & $X_{-15}+X_{-43}$ & $A_{2}$ \\
$6,7,8$ & 5 & $\left(X_{-16}\right)+\left(X_{-20}\right)+\left(X_{-29}\right)$ & $\left(3 A_{1}\right)^{\prime}$ \\
8 & 3 & $\left(X_{-16}\right)+\left(X_{-29}\right)+\left(X_{-41}\right)$ & $\left(3 A_{1}\right)^{\prime \prime}$ \\
3,5 & 2 & $\left(X_{-15}\right)+\left(X_{-16}\right)$ & $2 A_{1}$ \\
2 & 1 & $X_{-2}$ & $A_{1}$ \\
\hline
\end{tabular}

\section{Proof of the Correctness of the Diagram}

We shall write $i \rightarrow j$ if $i$ and $j$ are two nodes in the closure diagram (see Figures 5 and 6 ), with $i$ above $j$, that are joined by a line. We shall say that a pair $(i, j)$ is a critical pair if the following four conditions are satisfied:

(i) The node $i$ lies above the node $j$, i.e. $\operatorname{dim}\left(\mathcal{O}_{1}^{i}\right)>\operatorname{dim}\left(\mathcal{O}_{1}^{j}\right)$.

(ii) The diagram contains no downward path from $i$ to $j$.

(iii) If $\ell \rightarrow i$, then there is a downward path from $\ell$ to $j$.

(iv) If $j \rightarrow k$, then there is a downward path from $i$ to $k$.

We shall say that a critical pair $(i, j)$ is good if $\mathcal{O}_{1}^{i} \ngtr \mathcal{O}_{1}^{j}$. In order to complete the proof of the main theorem, it suffices to show that all critical pairs are good. It is tedious to find the critical pairs by inspection of the diagram. We used a short program to find all such pairs. The involution $\tilde{w}$ (see Section 2) permutes the set of critical pairs. Clearly, it suffices to consider just one critical pair from each of the $\tilde{w}$-orbits. In Table 5 we list the representatives of the $\tilde{w}$-orbits of critical pairs.

TABLE 5. Representatives of $\tilde{w}$-orbits of critical pairs

$\begin{array}{lllll}(7,3)^{*} & (17,3)^{\dagger} & (12,6) & (16,7) & (19,13)^{\dagger} \\ (22,13) & (23,13) & (39,16)^{\dagger} & (40,16) & (44,16) \\ (45,16) & (56,16)^{\dagger} & (24,20)^{*} & (30,22)^{*} & (32,22)^{\dagger} \\ (40,22)^{\dagger} & (39,26) & (31,27) & (60,30)^{*} & (62,30) \\ (56,35) & (53,38) & (44,39)^{*} & (45,39)^{*} & (55,39)^{\dagger} \\ (75,44)^{\dagger} & (68,51) & (77,55) & (69,62) & (71,66)^{*} \\ (79,72)^{\dagger} & (79,74)^{\dagger} & (88,76) & (85,80) & (84,82)^{*} \\ (86,82)^{\dagger} & (90,87)^{\dagger} & & & \end{array}$

By using the Mizuno's diagram (see [10, Figure 1]), it is easy to see that the eight critical pairs in Table 5 that are marked by an asterisk are good. For instance, the critical pair $(7,3)$ is good because $\mathcal{O}_{1}^{7} \subset \mathcal{O}^{5}, \mathcal{O}_{1}^{3} \subset \mathcal{O}^{3}$, and $\mathcal{O}^{5} \ngtr \mathcal{O}^{3}$. 
Let $\omega_{i}(1 \leq i \leq 7)$ be the fundamental weights of $R$ and let $V$ be the fundamental $\mathfrak{g}$-module with highest weight $\omega_{7}$. We make it into a $Z_{2}$-graded module as explained in the Appendix. The integers $d_{i}(j, k)$ defined there are recorded in Table 8. We now invoke [8, Theorem 4.1] to obtain a list of pairs $(i, j)$ such that $\operatorname{dim}\left(\mathcal{O}_{1}^{i}\right)>\operatorname{dim}\left(\mathcal{O}_{1}^{j}\right)$ and $\mathcal{O}_{1}^{i} \ngtr \mathcal{O}_{1}^{j}$. Some of these pairs (those that will be used in our proof) are listed in Table 6. By inspecting this table, we see that the 12 critical pairs in Table 5 that are marked by a dagger are good.

$\begin{array}{lllll}\text { TABLE } & \text { 6. Some pairs }(i, j) & \text { such that } \mathcal{O}_{1}^{i} \ngtr \mathcal{O}_{1}^{j} \\ (17,3) & (19,13) & (20,13) & (21,13) & (36,16) \\ (38,16) & (39,16) & (43,16) & (56,16) & (32,22) \\ (40,22) & (54,30) & (42,35) & (55,39) & (75,44) \\ (56,51) & (58,51) & (61,51) & (69,55) & (75,55) \\ (79,72) & (79,74) & (86,82) & (90,87) & \end{array}$

We are now faced with the most difficult part of the proof: To show that the 17 unadorned critical pairs in Table 5 are good. For that purpose we shall use some basic concepts from the theory of prehomogeneous vector spaces (PV's) [13, 14]. Unfortunately, the arguments are too long and tedious to be included in this paper. For that reason we shall give an outline of our method and then apply it to one typical critical pair.

\section{Outline of the Method}

Let us consider a fixed node, say $i$, in our diagram. We shall say that a node $j$ is a child of $i$ if $\mathcal{O}_{1}^{i}>\mathcal{O}_{1}^{j}$ and there is no $k$ such that $\mathcal{O}_{1}^{i}>\mathcal{O}_{1}^{k}>\mathcal{O}_{1}^{j}$. Let us also say that the node $i$ is good if it has the property that $i \rightarrow j$ if and only if $j$ is a child of $i$. (A posteriori, all nodes are good.)

The centralizer $Z=Z_{K^{0}}\left(H^{i}\right)$ is a connected reductive subgroup of $K^{0}$ which can be easily determined (up to local isomorphism) from the integers $\beta_{k}\left(H^{i}\right)$ given in Table 3. Furthermore, $Z$ is a Levi factor of the parabolic subgroup $Q_{H^{i}}$ of $K^{0}$ (defined in Section 2). The centralizer $M$ of $E^{i}$ in $Z$ is reductive, and consequently the PV $\left(Z, \mathfrak{g}_{H^{i}}(1,2)\right)$ is regular [13]. The intersection $\mathcal{O}_{1}^{i} \cap \mathfrak{g}_{H^{i}}(1,2)$ is the open dense $Z$-orbit in $\mathfrak{g}_{H^{i}}(1,2)$. Its complement, $S$, in $\mathfrak{g}_{H^{i}}(1,2)$ is a hypersurface. In the theory of PV's, $S$ is called the singular set. We note that the representative $E^{i}$ of $\mathcal{O}_{1}^{i}$ is a generic element of this PV. The set $S$ is a union of irreducible conical hypersurfaces $S_{k}$ defined by equations $f_{k}=0$, where the $f_{k}(k=1,2, \ldots, r)$ are the basic relative invariants of this PV. Recall that $f_{1}, \ldots, f_{r}$ are algebraically independent irreducible homogeneous polynomials on $\mathfrak{g}_{H^{i}}(1,2)$. One knows that $r \leq \ell$, where $\ell$ is the length of $\mathfrak{g}_{H^{i}}(1,2)$ as a $Z$-module. More precisely, $r=$ $\operatorname{dim}\left(Z / Z^{\prime} M\right)$ where $Z^{\prime}$ is the derived subgroup of $Z$ (see [14]).

The pair $\left(Q_{H^{i}}, \mathfrak{p}_{2}\left(H^{i}\right)\right)$ is also a PV and its singular set $\hat{S}$ is the union of the irreducible hypersurfaces $\hat{S}_{k}=S_{k}+\mathfrak{p}_{3}\left(H^{i}\right)$. For each $k$ extend the polynomial function $f_{k}: \mathfrak{g}_{H^{i}}(1,2) \rightarrow \mathbf{C}$ to the polynomial function $\hat{f}_{k}: \mathfrak{p}_{2}\left(H^{i}\right) \rightarrow \mathbf{C}$ by setting $\hat{f}_{k}(x+y)=f_{k}(x)$ for $x \in \mathfrak{g}_{H^{i}}(1,2)$ and $y \in \mathfrak{p}_{3}\left(H^{i}\right)$. These $\hat{f}_{k}$ are the basic relative invariants of $\left(Q_{H^{i}}, \mathfrak{p}_{2}\left(H^{i}\right)\right)$ and $\hat{S}_{k}$ is defined by the equation $\hat{f}_{k}=0$.

The basis of our method is the following result which was formulated after several discussions that I had with J. Sekiguchi. 
Proposition 7.1. Use the above notation. For each $k \in\{1,2, \ldots, r\}$ there exists a unique node $j_{k}$ in our diagram such that $\hat{S}_{k} \cap \mathcal{O}_{1}^{j_{k}}$ is a dense open subset of $\hat{S}_{k}$. Every child of $i$ belongs to the set $\left\{j_{1}, j_{2}, \ldots, j_{r}\right\}$.

Proof. Let $k$ be fixed. Since $\hat{S}_{k} \subset \mathfrak{p}_{2}\left(H^{i}\right) \subset \mathcal{N}_{1}$ and there are only finitely many $K^{0}$-orbits in $\mathcal{N}_{1}$, it follows that there exists a $j$ such that $\hat{S}_{k} \cap \mathcal{O}_{1}^{j}$ is dense in $\hat{S}_{k}$. For two different $K^{0}$-orbits, it is impossible that each of them be contained in the closure of the other. This implies that the above $j$ is unique and we denote it by $j_{k}$. If $E \in \hat{S}_{k} \backslash \mathcal{O}_{1}^{j_{k}}$, then $E \in \mathcal{O}_{1}^{m}$ for some $m \neq j_{k}$. Since $\hat{S}_{k} \cap \mathcal{O}_{1}^{j_{k}}$ is dense in $\hat{S}_{k}$, we have $\mathcal{O}_{1}^{j_{k}}>\mathcal{O}_{1}^{m}$. Consequently, the closure of $\hat{S}_{k} \cap \mathcal{O}_{1}^{m}$ is contained in $\hat{S}_{k} \backslash \mathcal{O}_{1}^{j_{k}}$. This shows that the set $\hat{S}_{k} \cap \mathcal{O}_{1}^{j_{k}}$ is open in $\hat{S}_{k}$. The first assertion is proved.

To prove the second assertion, let $j$ be a child of $i$. Since

$$
\overline{\mathcal{O}_{1}^{i}}=K^{0} \cdot \mathfrak{p}_{2}\left(H^{i}\right)=\mathcal{O}_{1}^{i} \cup\left(\bigcup_{k=1}^{r} K^{0} \cdot \hat{S}_{k}\right),
$$

we must have $\mathcal{O}_{1}^{j} \subset K^{0} \cdot \hat{S}_{k}$ for some $k$. Then $\mathcal{O}_{1}^{i}>\mathcal{O}_{1}^{j_{k}} \geq \mathcal{O}_{1}^{j}$ and, since $j$ is a child of $i$, we must have $\mathcal{O}_{1}^{j_{k}}=\mathcal{O}_{1}^{j}$, i.e., $j=j_{k}$.

To simplify notation, we set $\mathcal{P}_{k}=\hat{S}_{k} \cap \mathcal{O}_{1}^{j_{k}}$ for $1 \leq k \leq r$.

We now make several remarks concerning this proposition. First, it is clear from the definitions that $\mathcal{O}_{1}^{i}>\mathcal{O}_{1}^{j_{k}}$ for $1 \leq k \leq r$. Second, the nodes $j_{1}, j_{2}, \ldots, j_{r}$ are not necessarily distinct. Third, there exist examples such that $\mathcal{O}_{1}^{j_{k}}>\mathcal{O}_{1}^{j_{\ell}}$ for some $k$ and $\ell$. Then $j_{\ell}$ is not a child of $i$.

In most cases, the hypersurface $\hat{S}_{k}$ contains a dense open $Q_{H^{i} \text {-orbit which is }}$ necessarily contained in $\mathcal{P}_{k}$. In such cases it is not difficult to determine $j_{k}$. Once all the $j_{k}$ are computed, it is easy to identify all the children of the node $i$ and thus verify that $i$ is a good node.

\section{TREATEMENT OF A CRITICAL PAIR}

In this section we illustrate our method by proving that the critical pair $(62,30)$ is good. The centralizer $Z=Z_{K^{0}}\left(H^{62}\right)=\left(\mathrm{SL}_{2}\right)^{2} \cdot T_{5}$ has a 5 -dimensional central torus $T_{5}$. The simple roots of the two $\mathrm{SL}_{2}$ factors are $\left\{\alpha_{3}\right\}$ and $\left\{\alpha_{5}\right\}$. The space $\mathfrak{g}_{H^{62}}(1,2)$ is a direct sum of five simple $Z$-modules: the 4-dimensional $Z$-module $V(4)=\left\langle X_{-46}, X_{-43}, X_{-42}, X_{-38}\right\rangle$ on which both factors $\mathrm{SL}_{2}$ act non-trivially, the 2-dimensional $Z$-module $V(2)=\left\langle X_{52}, X_{55}\right\rangle$ on which the second $\mathrm{SL}_{2}$ factor acts trivially, the 2-dimensional $Z$-module $W(2)=\left\langle X_{-45}, X_{-41}\right\rangle$ on which the first $\mathrm{SL}_{2}$ factor acts trivially, and two 1-dimensional modules $V(1)=\left\langle X_{-39}\right\rangle$ and $W(1)=$ $\left\langle X_{54}\right\rangle$. The PV $\left(Z, \mathfrak{g}_{H^{62}}(1,2)\right)$ has only 4 basic relative invariants. Thus, using the notations from the previous section, we have $r=4$ and $\ell=5$.

For illustrative purposes we exhibit the weight diagram for this module in Figure 8 (all weights are simple). A vertex labeled $i$ stands for the one-dimensional weight space spanned by the root vector $X_{i}$. The action of the simple root vectors of the Lie algebra of $Z$ is indicated by the arrows. The involution $\tilde{w}$ fixes the characteristic $H^{62}$ and so leaves the space $\mathfrak{g}_{H^{62}}(1,2)$ invariant. More precisely, by using Table 1 we see that this involution leaves invariant the module $V(4)$, and interchanges the modules $V(2)$ and $W(2)$, and also $V(1)$ and $W(1)$.

Our representative of the orbit $\mathcal{O}_{1}^{62}$ from Table 3 is

$$
E^{62}=\left(X_{54}+X_{-45}+X_{-38}+X_{52}+X_{-39}\right)+\left(X_{-46}\right)
$$


and its type is $\left(A_{5}+A_{1}\right)^{\prime}$. This representative is a generic element of the PV $\left(Z, \mathfrak{g}_{H^{62}}(1,2)\right)$, i.e., $Z \cdot E^{62}$ is a dense open subset of $\mathfrak{g}_{H^{62}}(1,2)$. The six black nodes in Figure 8 depict the weights of the weight vectors that occur in the expression for $E^{62}$.
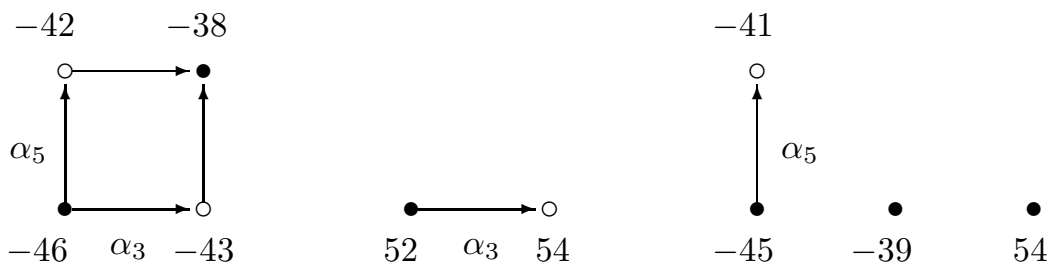

FiguRE 8 . The weight diagram of $\mathfrak{g}_{H^{62}}(1,2)$

Let us consider the following four vectors in $\mathfrak{p}_{2}\left(H^{62}\right)$ :

$$
\begin{aligned}
& E_{1}=X_{54}+X_{-45}+X_{-38}+X_{52}+X_{-39}, \\
& E_{2}=\left(X_{54}+X_{-45}+X_{-38}+X_{52}\right)+\left(X_{-46}\right), \\
& E_{3}=\left(X_{-45}+X_{-38}+X_{52}+X_{-39}\right)+\left(X_{-46}\right), \\
& E_{4}=\left(X_{54}+X_{-45}+X_{-38}\right)+\left(X_{-35}+X_{-39}+X_{55}+X_{-46}\right) .
\end{aligned}
$$

The vector $E_{1}$ is of type $\left(A_{5}\right)^{\prime}$, while $E_{2}$ and $E_{3}$ are of type $A_{4}+A_{1}$. The vector $E_{4}$ is not of type $A_{3}+A_{4}$; its diagram is shown in Figure 9.

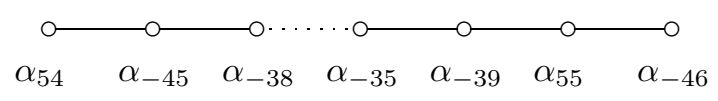

Figure 9. The type of $E_{4}$

From the proof that $62 \rightarrow 59$ (see Table 4 ) we see that $E_{1} \in \mathcal{O}_{1}^{59}$. By computing the characteristic $H$ of $E_{2}$, we can show that $E_{2} \in \mathcal{O}_{1}^{48}$. The details are as follows. By using [3, Chapter VIII, §7, Lemma 2], we have

$$
H=4 H_{-45}+6 H_{-38}+6 H_{52}+4 H_{-39}+H_{-46} .
$$

By using [10, Table 10], we obtain that

$$
H=-5 H_{1}-9 H_{2}-10 H_{3}-14 H_{4}-8 H_{5}-3 H_{6}-2 H_{7},
$$

and so

$$
\beta_{i}(H)=5,0,-1,-1,1,4,-1 \quad(1 \leq i \leq 7) .
$$

By transforming $H$ with a suitable element of the Weyl group of $(\mathfrak{k}, \mathfrak{h})$, we obtain the element $H^{\prime} \in \mathfrak{h}$ such that

$$
\beta_{i}\left(H^{\prime}\right)=3,1,0,1,0,2,1 \quad(1 \leq i \leq 7) .
$$

By looking up Table 3, we see that $H^{\prime}=H^{48}$ and, consequently, $E_{2} \in \mathcal{O}_{1}^{48}$. By applying the involution $\tilde{w}$, we infer that $E_{3} \in \mathcal{O}_{1}^{49}$. Finally, we show that $E_{4} \in \mathcal{O}_{1}^{54}$. The seven roots in Figure 9 are linearly independent. Consequently, the vector $E_{4}^{\prime}=E_{4}-X_{-38}$ belongs to the closure of the orbit $K^{0} \cdot E_{4}$. A computation shows that both orbits $K^{0} \cdot E_{4}$ and $K^{0} \cdot E_{4}^{\prime}$ have the same dimension, namely 53 . It 
follows that these orbits are the same. As $E_{4}^{\prime}$ is of type $A_{4}+A_{2}$, we conclude that $E_{4}, E_{4}^{\prime} \in \mathcal{O}_{1}^{54}$ (see Table 3 ).

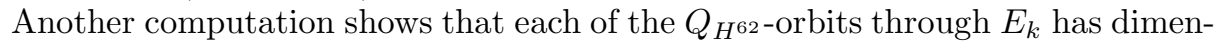
sion 28. Since $\mathfrak{p}_{2}\left(H^{62}\right)$ has dimension 29 , we conclude that the four hypersurfaces

$$
\hat{S}_{k}=\overline{Q_{H^{62}} \cdot E_{k}} \quad(k=1,2,3,4)
$$

are the irreducible components of the singular set $\hat{S}$ of $\left(Q_{H^{62}}, \mathfrak{p}_{2}\left(H^{62}\right)\right)$. It follows that $j_{1}=59, j_{2}=48, j_{3}=49$, and $j_{4}=54$ (using the notation from the previous section). Since $62 \rightarrow 59 \rightarrow 54 \rightarrow 48$ and $54 \rightarrow 49$, we conclude that the node 62 has only one child, namely 59 . Thus we have shown that the node 62 is good, i.e.,

$$
\overline{\mathcal{O}_{1}^{62}}=\mathcal{O}_{1}^{62} \cup \overline{\mathcal{O}_{1}^{59}}
$$

Next we have to examine the node 59. The centralizer $Z_{K^{0}}\left(H^{59}\right)=T_{7}$ is the maximal torus of $K^{0}$ (with Lie algebra $\mathfrak{h}$ ). The space $\mathfrak{g}_{H^{59}}(1,2)$ is a direct sum of five 1-dimensional $T_{7}$-modules:

$$
\left\langle X_{54}\right\rangle, \quad\left\langle X_{-45}\right\rangle, \quad\left\langle X_{-38}\right\rangle, \quad\left\langle X_{52}\right\rangle, \quad\left\langle X_{-39}\right\rangle .
$$

Hence, in this case, $r=\ell=5$. The involution $\tilde{w}$ fixes the characteristic $H^{59}$, thus leaving the space $\mathfrak{g}_{H^{59}}(1,2)$ invariant. By using Table 1 we see that this involution leaves invariant the module $\left\langle X_{-38}\right\rangle$, and interchanges the modules $\left\langle X_{54}\right\rangle$ and $\left\langle X_{-39}\right\rangle$, and also the modules $\left\langle X_{-45}\right\rangle$ and $\left\langle X_{52}\right\rangle$.

Let us write an arbitrary $X \in \mathfrak{p}_{2}\left(H^{59}\right)$ as

$$
X=x_{1} X_{54}+x_{2} X_{-45}+x_{3} X_{-38}+x_{4} X_{52}+x_{5} X_{-39}+X^{\prime},
$$

where $X^{\prime} \in \mathfrak{p}_{3}\left(H^{59}\right)$. The singular set $\hat{S}$ of the PV $\left(Q_{H^{59}}, \mathfrak{p}_{2}\left(H^{59}\right)\right)$ is the union of the five hyperplanes $\hat{S}_{k}: x_{k}=0(1 \leq k \leq 5)$.

Our representative of $\mathcal{O}_{1}^{59}$ from Table 3 is

$$
E^{59}=X_{54}+X_{-45}+X_{-38}+X_{52}+X_{-39}
$$

and its type is $\left(A_{5}\right)^{\prime}$. It is a generic element of the $\mathrm{PV}\left(T_{7}, \mathfrak{g}_{H^{59}}(1,2)\right)$.

Let us consider the following three vectors:

$$
\begin{aligned}
& E_{1}=\left(X_{-45}+X_{-38}+X_{52}+X_{-39}\right)+\left(X_{-29}\right) \in \hat{S}_{1}, \\
& E_{2}=\left(X_{-40}+X_{-39}+X_{52}+X_{-38}\right)+\left(X_{-41}+X_{54}\right) \in \hat{S}_{2}, \\
& E_{3}=\left(X_{-32}+X_{54}+X_{-45}\right)+\left(X_{52}+X_{-39}+X_{-35}\right)+X_{-40} \in \hat{S}_{3} .
\end{aligned}
$$

A computation shows that each of the $Q_{H^{59}}$-orbits through $E_{k}$ has dimension 25. Since $\mathfrak{p}_{2}\left(H^{59}\right)$ has dimension 26 , we conclude that

$$
\hat{S}_{k}=\overline{Q_{H^{59}} \cdot E_{k}} \quad(k=1,2,3) .
$$

The vector $E_{1}$ is of type $A_{4}+A_{1}$, while $E_{2}$ is of type $A_{4}+A_{2}$. It follows that $E_{1} \in \mathcal{O}_{1}^{48} \cup \mathcal{O}_{1}^{49}$ and $E_{2} \in \mathcal{O}_{1}^{54}$ (see Table 3 ). Hence $\hat{S}_{1}, \hat{S}_{2} \subset \overline{\mathcal{O}_{1}^{54}}$. By applying $\tilde{w}$, 
we infer that also $\hat{S}_{4}, \hat{S}_{5} \subset \overline{\mathcal{O}_{1}^{54}}$. The vector $E_{3}$ is not of type $2 A_{3}+A_{1}$; its diagram is shown in Figure 10.

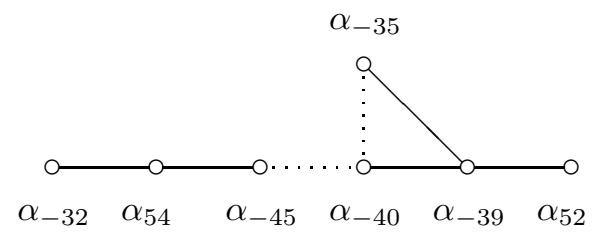

Figure 10. The type of $E_{3}$

We also claim that $\hat{S}_{3} \subset \overline{\mathcal{O}_{1}^{54}}$. The seven roots in Figure 10 are linearly independent. Consequently, the vector $E_{3}^{\prime}=E_{3}-X_{-40}$ belongs to the closure of the orbit $K^{0} \cdot E_{3}$. A computation shows that both orbits $K^{0} \cdot E_{3}$ and $K^{0} \cdot E_{3}^{\prime}$ have the same dimension, namely 50. It follows that these orbits are the same. As $E_{3}^{\prime}$ is of type $2 A_{3}$, we conclude that $E_{3}, E_{3}^{\prime} \in \mathcal{O}_{1}^{43}$ (see Table 3 ). As $\mathcal{O}_{1}^{54}>\mathcal{O}_{1}^{43}$ (see the closure diagram), our claim is proven.

In summary, we have shown that all five hyperplanes $\hat{S}_{k}$ are contained in $\overline{\mathcal{O}_{1}^{54}}$. This implies that the node 59 is good, i.e.,

$$
\overline{\mathcal{O}_{1}^{59}}=\mathcal{O}_{1}^{59} \cup \overline{\mathcal{O}_{1}^{54}}
$$

Since $\mathcal{O}_{1}^{54} \ngtr \mathcal{O}_{1}^{30}$ (see Table 6), the equalities (8.1) and (8.2) imply that $\mathcal{O}_{1}^{62} \ngtr \mathcal{O}_{1}^{30}$. Hence we have shown that the critical pair $(62,30)$ is good.

\section{Appendix}

In Table 7 we list, for each $i$, the indices $k$ of the roots $\alpha_{k}$ for which $\mathfrak{g}^{\alpha_{k}} \subset \mathfrak{p}_{2}\left(H^{i}\right)$. We list first those $k$ for which $\mathfrak{g}^{\alpha_{k}} \subset \mathfrak{g}_{H^{i}}(1,2)$ and separate them by a semicolon from the $k$ 's for which $\mathfrak{g}^{\alpha_{k}} \subset \mathfrak{p}_{3}\left(H^{i}\right)$. 
TABLE 7. Root spaces in $\mathfrak{p}_{2}\left(H^{i}\right)\left(\mathfrak{g}_{0}=\mathrm{E}\right.$ V)

\begin{tabular}{|c|c|}
\hline$i$ & $\mathfrak{p}_{2}\left(H^{i}\right)$ \\
\hline 1 & -2 \\
\hline 2 & $-28,-22,-16,-15,-9,-2$ \\
\hline 3 & $-49,-45,-41,-40,-36,-35,-30,-29,-28,-23,-22,-16,-15,-9,-2$; \\
\hline 4 & $49,52,-37,55,-32,57,-28,-26,59,-22,-20,-16,-15,-9,-2$ \\
\hline 5 & $-33,-29,-26,-23,-22,-20,-16,-15,-9 ;-2$ \\
\hline 6 & $\begin{array}{l}-50,-46,-43,-42,-40,-38,-37,-35,-33,-32,-29,-28,-26,-23 \\
-22,-20,-16,-15,-9,-2\end{array}$ \\
\hline 7 & $\begin{array}{l}50,-39,54,-36,-33,57,-30,-29,-26,59,-23,-22,-20,-16,-15,-9 \\
-2\end{array}$ \\
\hline 8 & $-20,-41,-36,-35,-30,-29,-28,-23,-22,-16 ;-15,-9,-2$ \\
\hline 9 & $-23,55,-32,57,-28,-26,59,-22,-20,-15 ;-16,-9,-2$ \\
\hline 10 & $\begin{array}{l}-30,-43,-40,-38,-35,-33,-32,-29,-28,-26,-22,-20,-15 \\
-23,-16,-9,-2\end{array}$ \\
\hline 11 & $\begin{array}{l}-42,-38,-37,-35,-33,-32,-29,-28,-26,-23,-22,-16,59 \\
-20,-15,-9,-2\end{array}$ \\
\hline 12 & $-28,-36,-30,-29,-23,57,-26,59,-20 ;-22,-16,-15,-9,-2$ \\
\hline 13 & $\begin{array}{l}-47,-44,-42,-41,-39,-38,-37,-36,-35,-33,-32,-30,-29,-28, \\
-26,-23,-22,-16 ;-20,-15,-9,-2\end{array}$ \\
\hline 14 & $\begin{array}{l}47,-43,51,-40,-38,54,55,-35,-33,-32,57,-29,-28,-26,59 \\
-22,-20,-15 ;-23,-16,-9,-2\end{array}$ \\
\hline 15 & $\begin{array}{l}-30,-38,-35,-33,-32,-29,-28,-26,-22,59 \\
-20,-15,-23,-16,-9,-2\end{array}$ \\
\hline 16 & $\begin{array}{l}-59,-57,-55,-54,-52,-51,-50,-49,-48,-47,-46,-45,-44,-43, \\
-42,-41,-40,-39,-38,-37,-36,-35,-33,-32,-30,-29,-28,-26, \\
-23,-22,-20,-16,-15,-9,-2\end{array}$ \\
\hline 17 & $\begin{array}{l}30,36,39,41,-50,44,45,-46,47,48,49,-43,-42,51,52,-40,-38,-37 \\
54,55,-35,-33,-32,57,-29,-28,-26,59,-23,-22 \\
-20,-16,-15,-9,-2\end{array}$ \\
\hline 18 & $\begin{array}{l}-47,-44,-42,-41,-39,-38,-37,-36,-35,-33,-32,-30,-29,-28, \\
-26,-23,-22,-16,59 ;-20,-15,-9,-2\end{array}$ \\
\hline 19 & $\begin{array}{l}-30,47,-43,51,-40,-38,54,55,-35,-33,-32,57,-29 \\
-28,-26,59,-22,-20,-15 ;-23,-16,-9,-2\end{array}$ \\
\hline 20 & $\begin{array}{l}-28,50,-39,54,-33 \\
-36,-30,-29,-23,57,-26,59,-20,-22,-16,-15,-9,-2\end{array}$ \\
\hline 21 & $\begin{array}{l}-45,-41,-40,-36,-35,-30,-29,-23,52,-37,55,-32,57 \\
-26,59,-20 ;-28,-22,-16,-15,-9,-2\end{array}$ \\
\hline 22 & $\begin{array}{l}-49,-45,-41,-40,-35,-28,50,-39,54,-33,57,-26,59,-20 \\
-36,-30,-29,-23,-22,-16,-15,-9,-2\end{array}$ \\
\hline 23 & $\begin{array}{l}49,52,-37,55,-32,-28,50,-39,54,-36,-33,-30,-29,-23 \\
57,-26,59,-22,-20,-16,-15,-9,-2\end{array}$ \\
\hline 24 & $\begin{array}{l}-33,-36,-30,-37,-32,-40,-35,57,59 \\
-26,-20,-28,-29,-23,-22,-16,-15,-9,-2\end{array}$ \\
\hline 25 & $\begin{array}{l}-38,-35,-32,-28,-39,-36,54,57 \\
-30,-33,-29,-26,-22,59,-20,-15,-23,-16,-9,-2\end{array}$ \\
\hline
\end{tabular}


TABle 7. (continued)

\begin{tabular}{|c|c|}
\hline$i$ & $\overline{\mathfrak{p}_{2}\left(H^{i}\right)}$ \\
\hline 26 & $\begin{array}{l}-39,-36,-30,-46,-43,-42,-40,-38,-37,-35,-32, \\
-33,-29,-26,-23,-22,-20,-16,-15,-9,-2\end{array}$ \\
\hline 27 & $\begin{array}{l}46,-44,50,51,-41,-39,-38,54,55,-36,-35,-33,-32,57,-29 \\
-28,-26,-22 ;-30,-23,-16,59,-20,-15,-9,-2\end{array}$ \\
\hline 28 & $\begin{array}{l}-32,-39,-33,-45,-41,-40,-35,57,59 \\
-26,-20,-28,-36,-29,-22,-15,-30,-23,-16,-9,-2\end{array}$ \\
\hline 29 & $\begin{array}{l}-35,-36,-30,52,-37,55,-32,54,-33 \\
-28,-29,-23,57,-26,-22,-16,59,-20,-15,-9,-2\end{array}$ \\
\hline 30 & $\begin{array}{l}-46,-43,-42,-40,-38,-37,-35,-32,-28,50 ;-39,-36,-30,54,57, \\
59,-33,-29,-26,-23,-22,-20,-16,-15,-9,-2\end{array}$ \\
\hline 31 & $\begin{array}{l}-39,-40,-41,-42,-38,-37,-32,59 \\
-33,-26,-35,-28,-36,-30,-20,-29,-23,-22,-16,-15,-9,-2\end{array}$ \\
\hline 32 & $\begin{array}{l}-30,-37,-43,-40,-38,-35,54,55 \\
-32,-28,-33,-29,57,59,-23,-26,-22,-20,-15,-16,-9,-2\end{array}$ \\
\hline 33 & $\begin{array}{l}-40,-41,-36,51,-38,54,55,-33,-32,57,-26 \\
-35,-29,-28,-22,59,-20,-15,-30,-23,-16,-9,-2\end{array}$ \\
\hline 34 & $\begin{array}{l}-37,-44,-41,-39,-38,-36,-35,-33,-29,55,57 \\
-30,-23,-32,-28,-26,-22,-16,59,-20,-15,-9,-2\end{array}$ \\
\hline 35 & $\begin{array}{l}-48,-45,-44,-43,-41,-40,-39,-38,-36,-35,-33,-29,55,57,59 \\
-32,-28,-26,-22,-20,-15,-30,-23,-16,-9,-2\end{array}$ \\
\hline 36 & $\begin{array}{l}-41,-36,-30,48,-42,51,52,-38,-37,54,55,-33,-32,57,-26 \\
-35,-29,-28,-23,-22,-16,59,-20,-15,-9,-2\end{array}$ \\
\hline 37 & $\begin{array}{l}-37,-38,-33,-40,-41,-36,55,57 \\
-30,-32,-26,-35,-29,59,-20,-23,-28,-22,-15,-16,-9,-2\end{array}$ \\
\hline 38 & $\begin{array}{l}-45,-41,-36,-30,-50,-46,-43,-42,-38,-33,52,55,57,59 \\
-37,-32,-26,-20,-40,-35,-29,-23,-28,-22,-16,-15,-9,-2\end{array}$ \\
\hline 39 & $\begin{array}{l}37,-52,42,-49,-48,46,47,-45,-44,-43,50,51,-41,-40,-39,-38,54 \\
55,-36,-35,-33,-32,57,-29,-28,-26,59,-22,-20,-15 \\
-30,-23,-16,-9,-2\end{array}$ \\
\hline 40 & $\begin{array}{l}40,43,45,-47,46,48,49,-44,-42,50,51,52,-41,-39,-38,-37,54,55 \\
-36,-35,-33,-32,57,-30,-29,-28,-26,-23,-22,-16 \\
59,-20,-15,-9,-2\end{array}$ \\
\hline 41 & $\begin{array}{l}-37,-48,-45,-44,-43,-41,-40,-39,-38,-36,-35,-33,-29 \\
55,57,59 ;-30,-23,-32,-28,-26,-22,-20,-15,-16,-9,-2\end{array}$ \\
\hline 42 & $\begin{array}{l}-40,-41,-36,-30,48,-42,51,52,-38,-37,54,55,-33,-32,57,-26 \\
-35,-29,-28,-23,-22,-16,59,-20,-15,-9,-2\end{array}$ \\
\hline 43 & $\begin{array}{l}-45,-41,-40,-35,50,-39,54,-33,52,-37,55,-32 \\
-28,-36,-30,-29,-23,57,-26,59,-20,-22,-16,-15,-9,-2\end{array}$ \\
\hline 44 & $\begin{array}{l}-49,50,-39,54,-33,52,-37,55,-32 ;-45,-41,-40,-35,57,-26,59 \\
-20,-28,-36,-30,-29,-23,-22,-16,-15,-9,-2\end{array}$ \\
\hline 45 & $\begin{array}{l}-45,-41,-40,-35,49,50,-39,54,-33 ;-36,-30,-29,-23,52,-37,55 \\
-32,-28,57,-26,59,-20,-22,-16,-15,-9,-2\end{array}$ \\
\hline 46 & $\begin{array}{l}-40,-41,-42,-38,-37,-32,50 ;-35,-28,54,57,-39,59,-33,-26,-36, \\
-30,-20,-29,-23,-22,-16,-15,-9,-2\end{array}$ \\
\hline 47 & $\begin{array}{l}-37,-43,-40,-38,-35,50,55 ;-32,-28,-39,-36,-30,54,-33,-29, \\
57,59,-23,-26,-22,-20,-15,-16,-9,-2\end{array}$ \\
\hline
\end{tabular}


TABle 7 . (continued)

\begin{tabular}{|c|c|}
\hline$t$ & $\mathfrak{p}_{2}\left(H^{i}\right)$ \\
\hline 48 & $\begin{array}{l}-39,-45,-41,-46,-43,-42,-38,57,59 ;-33,-36,-30,-40,-35, \\
-29,-23,-37,-32,-26,-20,-28,-22,-16,-15,-9,-2\end{array}$ \\
\hline 49 & $\begin{array}{l}-36,-30,-46,-43,-42,-38,52,55,54 ;-33,-37,-32,57,59,-26,-20 \\
-40,-35,-28,-29,-23,-22,-16,-15,-9,-2\end{array}$ \\
\hline 50 & $\begin{array}{l}-37,-38,-39,-40,-41,54,55 ;-32,-33,-35,-36,57,-26,-28,-29, \\
-30,59,-20,-22,-23,-15,-16,-9,-2\end{array}$ \\
\hline 51 & $\begin{array}{l}-37,-48,-45,-44,-43,-41,-40,-38,-35,50,54 ;-32,-28,57,59 \\
-39,-36,-33,-29,-26,-22,-20,-15,-30,-23,-16,-9,-2\end{array}$ \\
\hline 52 & $\begin{array}{l}-40,48,-42,51,52,-38,-37,55,-32,50,-39 ;-35,-28,-36,-30,54 \\
-33,57,-26,-29,-23,-22,-16,59,-20,-15,-9,-2\end{array}$ \\
\hline 53 & $\begin{array}{l}-37,-38,-40,-41,50,55 ;-32,-35,-39,54,-28,-33,-36,57,-26 \\
-29,-30,59,-20,-22,-23,-15,-16,-9,-2\end{array}$ \\
\hline 54 & $\begin{array}{l}-42,-37,-43,-40,-44,-41,-39,-36,51,54,55,57 ;-30,-38,-35 \\
-33,-32,-29,-28,-26,-22,59,-20,-15,-23,-16,-9,-2\end{array}$ \\
\hline 55 & $\begin{array}{l}50,54,57,59,-55,-52,-51,-49,-48,-47,-46,-45,-44,-43,-42,-41, \\
-40,-38,-37,-35,-32,-28 \\
-39,-36,-33,-30,-29,-26,-23,-22,-20,-16,-15,-9,-2\end{array}$ \\
\hline 56 & $\begin{array}{l}41,44,45,-46,47,48,49,-43,-42,51,52,-40,-38,-37,55,-35,-32, \\
-28,50,-39,-36,-30 \\
54,-33,57,-29,-26,59,-23,-22,-20,-16,-15,-9,-2\end{array}$ \\
\hline 57 & $\begin{array}{l}-37,-48,-45,-44,-43,-41,-40,-38,-35,50,54,55 ;-32,-28,-39, \\
-36,-33,-29,57,59,-26,-22,-20,-15,-30,-23,-16,-9,-2\end{array}$ \\
\hline 58 & $\begin{array}{l}-40,-41,48,-42,51,52,-38,-37,55,-32,50,-39 ;-35,-28,-36 \\
-30,54,-33,57,-26,-29,-23,-22,-16,59,-20,-15,-9,-2\end{array}$ \\
\hline 59 & $\begin{array}{l}-38,-39,-45,52,54 ;-33,-37,-40,-41,55,-32,-35,-36,57,-26,-29, \\
-30,59,-20,-23,-28,-22,-15,-16,-9,-2\end{array}$ \\
\hline 60 & $\begin{array}{l}-37,-38,-39,-49,54,55 ;-32,-33,-45,57,-26,-40,-41,59,-20 \\
-35,-36,-28,-29,-30,-22,-23,-15,-16,-9,-2\end{array}$ \\
\hline 61 & $\begin{array}{l}-38,-39,-40,-41,49,54 ;-33,-35,-36,52,-29,-30,-37,55,-23 \\
-32,57,-26,-28,59,-20,-22,-15,-16,-9,-2\end{array}$ \\
\hline 62 & $\begin{array}{l}-39,-45,-41,-46,-43,-42,-38,52,55,54 ;-33,-36,-30,-37,-32 \\
-40,-35,57,59,-26,-20,-28,-29,-23,-22,-16,-15,-9,-2\end{array}$ \\
\hline 63 & $\begin{array}{l}-45,-40,46,-44,50,51,-39,-38,54,-33,52,-37 ;-41,-36,-35,-29, \\
55,-32,57,-26,-28,-22,-30,-23,59,-20,-15,-16,-9,-2\end{array}$ \\
\hline 64 & $\begin{array}{l}-42,-37,-44,-39,-49,-45,51,54,55,57 ;-38,-33,-32,-26,-40 \\
-41,-36,59,-20,-30,-35,-29,-28,-22,-23,-16,-15,-9,-2\end{array}$ \\
\hline 65 & $\begin{array}{l}-43,-40,-44,-41,-39,-36,49,52,51,54 ;-37,-38,-35,-33,-29, \\
-30,55,57,-23,-32,-28,-26,-22,59,-20,-15,-16,-9,-2\end{array}$ \\
\hline 66 & $\begin{array}{l}-45,-41,-46,-43,-42,-38,50,52,55 ;-37,-32,-39,-40,-35,54 \\
-28,-33,-36,-30,57,59,-26,-20,-29,-23,-22,-16,-15,-9,-2\end{array}$ \\
\hline 67 & $\begin{array}{l}-42,-37,-43,-44,-39,-49,-45,51,54,55,57 ;-38,-33,-32,-26, \\
-40,-41,-36,59,-20,-30,-35,-29,-28,-22,-15,-23,-16,-9,-2\end{array}$ \\
\hline 68 & $\begin{array}{l}-42,-43,-40,-44,-41,-39,-36,49,52,51,54 ;-30,-37,-38,-35 \\
-33,-29,55,57,-23,-32,-28,-26,-22,59,-16,-20,-15,-9,-2\end{array}$ \\
\hline
\end{tabular}


TABLE 7. (continued)

\begin{tabular}{|c|c|}
\hline$\imath$ & $\mathfrak{p}_{2}\left(H^{i}\right)$ \\
\hline 69 & $\begin{array}{l}43,-47,46,48,-44,-42,50,51,52,-39,-38,-37,54,55,-33,-32,57, \\
-26,-49,-45,-40 ; \\
-41,-36,-35,-30,-29,-28,-23,-22,-16,59,-20,-15,-9,-2\end{array}$ \\
\hline 70 & $\begin{array}{l}42,-48,46,47,-45,-44,-43,50,51,-41,-40,-39,-38,54,-36,-35 \\
-33,-29,49,52,-37 \\
-30,-23,55,-32,57,-28,-26,59,-22,-20,-15,-16,-9,-2\end{array}$ \\
\hline 71 & $\begin{array}{l}-42,-43,-44,-39,-45,51,54,52 ;-37,-38,-33,-40,-41,-36,55,57 \\
-30,-32,-26,-35,-29,59,-20,-23,-28,-22,-15,-16,-9,-2\end{array}$ \\
\hline 72 & $\begin{array}{l}-42,-43,-40,-44,-41,49,52,50 ;-38,-35,54,55,-37,-39,-36,-30 \\
-32,-28,-33,-29,57,-23,59,-26,-22,-16,-20,-15,-9,-2\end{array}$ \\
\hline 73 & $\begin{array}{l}-42,-37,-43,-49,-45,50,51,55 ;-38,-32,-39,-41,-40,54,57,-33 \\
-26,-35,-28,-36,59,-20,-30,-29,-22,-15,-23,-16,-9,-2\end{array}$ \\
\hline 74 & $\begin{array}{l}-42,-43,-44,-45,50,55 ;-38,-40,-41,54,-35,-37,-32,-39,57 \\
-28,-33,-36,59,-29,-30,-23,-26,-20,-22,-15,-16,-9,-2\end{array}$ \\
\hline 75 & $\begin{array}{l}-41,-42,-43,50,51,52 ;-37,-38,-39,55,-32,-40,-35,-36,54 \\
-28,-30,-33,57,-26,59,-20,-29,-22,-23,-15,-16,-9,-2\end{array}$ \\
\hline 76 & $\begin{array}{l}-42,-37,-43,-44,-49,-45,50,51,55 ;-38,-32,-39,-40,-41,54,57 \\
-33,-26,-35,-28,-36,59,-20,-29,-22,-30,-15,-23,-16,-9,-2\end{array}$ \\
\hline 77 & $\begin{array}{l}-42,-43,-40,-44,-41,49,52,50,51 ;-37,-38,-35,-39,-36,54,55 \\
-30,-32,-28,-33,-29,57,-23,-26,-22,59,-16,-20,-15,-9,-2\end{array}$ \\
\hline 78 & $\begin{array}{l}49,52,55,50,54,-51,-48,-47,-46,-45,-44,-43,-42,-41,-40,-38 \\
-35 ;-37,-32,-28,-39,-36,-33,-30,-29,-23,57,59,-26,-22,-20 \\
-16,-15,-9,-2\end{array}$ \\
\hline 79 & $\begin{array}{l}44,-46,47,48,-43,-42,51,52,-38,-37,55,-32,-49,-45,-41,50,-39 \\
-36,-30,-40,-35,-28,54,-33,57,-26,59,-20,-29,-23,-22 \\
-16,-15,-9,-2\end{array}$ \\
\hline 80 & $\begin{array}{l}-44,-39,-45,-50,-46,51,54,52 \\
-41,-36,-42,-43,55,57,-30,-37,-38,-33,-40,59,-32,-26,-35 \\
-29,-20,-23,-28,-22,-15,-16,-9,-2\end{array}$ \\
\hline 81 & $\begin{array}{l}-42,-43,-44,-45,50,51,52 ;-37,-38,-39,-40,-41,54,55,-32,-33 \\
-35,-36,57,-26,-28,-29,-30,59,-20,-22,-23,-15,-16,-9,-2\end{array}$ \\
\hline 82 & $\begin{array}{l}-42,-43,-44,-49,50,52 ;-38,54,55,-37,-39,-45,-32,-33,-40,-41, \\
57,-35,59,-26,-36,-20,-28,-29,-30,-23,-22,-15,-16,-9,-2\end{array}$ \\
\hline 83 & $\begin{array}{l}-42,-43,-45,49,50,51 ;-38,-39,-41,-40,52,54,-33,-35,-36,-37 \\
55,-30,-32,-29,57,-23,-26,-28,59,-20,-22,-15,-16,-9,-2\end{array}$ \\
\hline 84 & $\begin{array}{l}-44,-45,-46,50,51,52 ;-41,-42,-43,55,-37,-38,-39,-40,54,-32, \\
-35,-36,57,-28,-30,-33,59,-26,-29,-20,-22,-23,-15,-16,-9,-2\end{array}$ \\
\hline 85 & $\begin{array}{l}-42,-43,-44,-49,50,51,52 ;-37,-38,-39,-45,54,55,-32,-33,-40 \\
-41,57,-26,-35,-36,59,-20,-28,-29,-30,-22,-23,-15,-16,-9,-2\end{array}$ \\
\hline 86 & $\begin{array}{l}-42,-43,-44,-45,49,50,51 ;-38,-39,-40,-41,52,54,-33,-35,-36, \\
-37,55,-29,-30,-32,57,-23,-26,-28,59,-20,-22,-15,-16,-9,-2\end{array}$ \\
\hline 87 & $\begin{array}{l}-49,50,54,-51,-48,-47,-46,-44,-43,-42,-38,52,55 \\
-37,-32,-39,-33,-45,-41,-40,-35,57,59,-26,-20,-28,-36 \\
-30,-29,-23,-22,-16,-15,-9,-2\end{array}$ \\
\hline
\end{tabular}


TABLE 7. (continued)

\begin{tabular}{|c|l|}
\hline$i$ & \multicolumn{1}{|c|}{$\mathfrak{p}_{2}\left(H^{i}\right)$} \\
\hline 88 & $44,-46,47,48,-43,-42,51,-38,-45,-41,49,50,-39 ;$ \\
& $-36,-30,-40,-35,52,-37,55,-32,54,-33,-28,-29,-23,57,-26$, \\
& $59,-20,-22,-16,-15,-9,-2$ \\
89 & $-46,-43,-47,-44,48,51,52,55,-49,-45,50 ;$ \\
& $-39,-40,-41,-42,-38,-37,-32,54,57,-33,-26,-35,-28,-36$, \\
& $-30,59,-20,-29,-23,-22,-16,-15,-9,-2$ \\
90 & $-46,-42,47,51,-48,-45,-44,-41,49,52,50 ;$ \\
& $-37,-39,-36,-43,-40,-38,-35,54,55,-30,-32,-28,-33,-29,57$, \\
91 & $59,-23,-26,-22,-20,-15,-16,-9,-2$ \\
& $-46,-42,47,51,-48,-44,-49,50,52 ;$ \\
& $-37,-39,-43,-38,-45,-41,54,55,-32,-33,-36,-40,-35,57,59$, \\
92 & $-26,-20,-28,-29,-30,-22,-15,-23,-16,-9,-2$ \\
& $-45,-46,-43,-47,-44,48,51,49,50 ;$ \\
& $-39,-40,-41,-42,-38,52,55,54,-33,-35,-36,-30,-37,-32$, \\
93 & $-26,-28,-29,-23,59,-20,-22,-16,-15,-9,-2$ \\
& $-46,-47,-48,-49,50,51,52 ;$ \\
& $-42,-43,-44,-45,54,55,-37,-38,-39,-40,-41,57,-32,-33,-35$, \\
94 & $-36,59,-26,-28,-29,-30,-20,-22,-23,-15,-16,-9,-2$ \\
& $-44,-45,-46,47,48,49,50 ;$ \\
& $-39,-41,-42,-43,51,52,-36,-37,-38,-40,54,55,-30,-32$, \\
& $-33,-35,57,-26,-28,-29,59,-20,-22,-23,-15,-16,-9,-2$ \\
\hline
\end{tabular}

Let $V$ be the 56-dimensional simple $\mathfrak{g}$-module with highest weight $\omega_{7}$ (one of the fundamental weights). It can be equipped with the $Z_{2}$-grading such that $V=$ $V_{0} \oplus V_{1}, \operatorname{dim} V_{0}=\operatorname{dim} V_{1}=28$, and the highest weight vector belongs to $V_{0}$. Thus

$$
\mathfrak{k} \cdot V_{i} \subset V_{i}, \mathfrak{p} \cdot V_{i} \subset V_{1-i}
$$

holds for $i=0,1$. We introduce the integers

$$
d_{i}(j, k)=\operatorname{dim}\left(V_{i} \cap \operatorname{ker} \rho\left(E^{k}\right)^{j}\right)
$$

where $i=0,1 ; j \geq 1 ; 1 \leq k \leq 94$, and $\rho$ is the representation of $\mathfrak{g}$ on $V$. ( $E^{k}$ is a representative of $\mathcal{O}_{1}^{k}$ from Table 3.) These integers are easy to compute using the $\mathfrak{s l}_{2}$-theory (see [8]) and are displayed in Table 8. 
TABLE 8 . The integers $d_{i}(j, k)$ for the module $V\left(\omega_{7}\right)$

\begin{tabular}{|c|c|c|}
\hline$k$ & $d_{0}(j, k) ; j=1,2, \ldots$ & $d_{1}(j, k) ; j=1,2, \ldots$ \\
\hline 1 & 2228 & 2228 \\
\hline 2 & 182728 & 182728 \\
\hline 3 & 14272728 & 132728 \\
\hline 4 & 132728 & 14272728 \\
\hline 5 & 162528 & 162528 \\
\hline 6 & 162228 & 162228 \\
\hline 7 & 162228 & 162228 \\
\hline 8 & 14242728 & 132428 \\
\hline 9 & 132428 & 14242728 \\
\hline 10 & 14222628 & 122228 \\
\hline 11 & 122228 & 14222628 \\
\hline 12 & 13222728 & 13222728 \\
\hline 13 & 14212428 & 102128 \\
\hline 14 & 102128 & 14212428 \\
\hline 15 & 12212628 & 12212628 \\
\hline 16 & 14212128 & 72128 \\
\hline 17 & 72128 & 14212128 \\
\hline 18 & 11212428 & 10212528 \\
\hline 19 & 10212528 & 11212428 \\
\hline 20 & 1217222728 & 1217222728 \\
\hline 21 & 1018262728 & 1018262728 \\
\hline 22 & $991722 \quad 272728$ & 817222728 \\
\hline 23 & 817222728 & 91722272728 \\
\hline 24 & 1018242728 & 1018242728 \\
\hline 25 & 1017222628 & 1017222628 \\
\hline 26 & 1016222528 & 1016222528 \\
\hline 27 & 1016222528 & 1016222528 \\
\hline 28 & 91622262728 & 816222628 \\
\hline 29 & 816222628 & 991622262728 \\
\hline 30 & $1013 \quad 161922 \quad 2528$ & 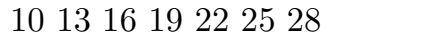 \\
\hline 31 & 91621252728 & 716222528 \\
\hline 32 & 716222528 & 91621252728 \\
\hline 33 & 81622252728 & 816212528 \\
\hline 34 & 816212528 & 81622252728 \\
\hline 35 & 81522252628 & 815202528 \\
\hline 36 & 815202528 & 81522252628 \\
\hline 37 & 81521252728 & 81521252728 \\
\hline 38 & 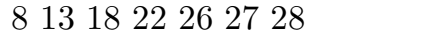 & $\begin{array}{lllllll}8 & 13 & 18 & 22 & 26 & 27 & 28\end{array}$ \\
\hline 39 & 71522252528 & 815182528 \\
\hline 40 & 815182528 & 71522252528 \\
\hline 41 & 81520252628 & 71520252728 \\
\hline 42 & 71520252728 & 81520252628 \\
\hline 43 & 8131822262728 & 8131822262728 \\
\hline 44 & 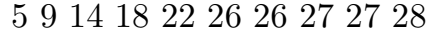 & 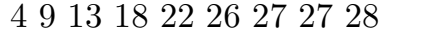 \\
\hline 45 & 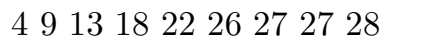 & 591418222626272728 \\
\hline
\end{tabular}


TABle 8. (continued)

\begin{tabular}{|c|c|c|}
\hline$k$ & $d_{0}(j, k) ; j=1,2, \ldots$ & $d_{1}(j, k) ; j=1,2, \ldots$ \\
\hline 46 & $8 \begin{array}{llllllll}8 & 13 & 16 & 19 & 22 & 25 & 27 & 28\end{array}$ & $\begin{array}{lllllll}7 & 13 & 16 & 19 & 22 & 25 & 28\end{array}$ \\
\hline 47 & 7131619222528 & 813161922252728 \\
\hline 48 & 8131822242728 & 6131822262728 \\
\hline 49 & 6131822262728 & 8131822242728 \\
\hline 50 & 7131822252728 & 7131822252728 \\
\hline 51 & 812161922252628 & 6121619222528 \\
\hline 52 & 6121619222528 & 812161922252628 \\
\hline 53 & 712161922252728 & 712161922252728 \\
\hline 54 & 6121821242628 & 6121821242628 \\
\hline 55 & 812151922252528 & 4121619222528 \\
\hline 56 & 4121619222528 & 812151922252528 \\
\hline 57 & 612161922252628 & 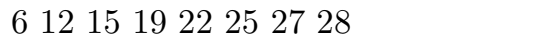 \\
\hline 58 & 612151922252728 & 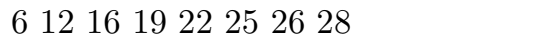 \\
\hline 59 & 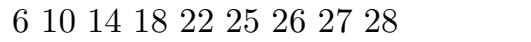 & 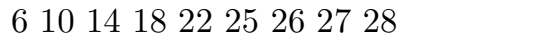 \\
\hline 60 & 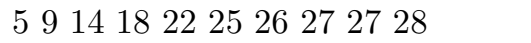 & 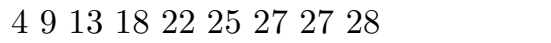 \\
\hline 61 & 4913182225272728 & 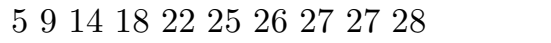 \\
\hline 62 & 61014182224262728 & 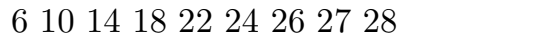 \\
\hline 63 & 61014182224262728 & 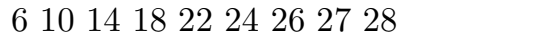 \\
\hline 64 & 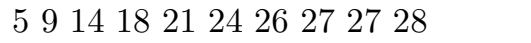 & 4913182224262728 \\
\hline 65 & 4913182224262728 & 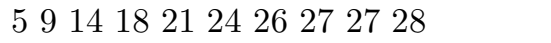 \\
\hline 66 & 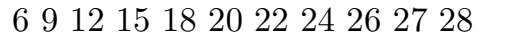 & 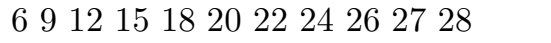 \\
\hline 67 & 591418212425272728 & 4913182124262728 \\
\hline 68 & 4913182124262728 & 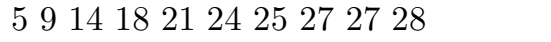 \\
\hline 69 & 591418202426272728 & 4913182224252728 \\
\hline 70 & 4913182224252728 & 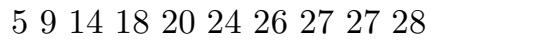 \\
\hline 71 & 48121518212425262728 & 48121518212425262728 \\
\hline 72 & 48121517202224262728 & 4812151820222426272728 \\
\hline 73 & 4812151820222426272728 & 48121517202224262728 \\
\hline 74 & 59121518202224252728 & 49121518202224262728 \\
\hline 75 & 49121518202224262728 & 59121518202224252728 \\
\hline 76 & 4812151820222425272728 & 48111517202224262728 \\
\hline 77 & 48111517202224262728 & 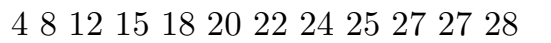 \\
\hline 78 & 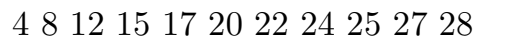 & 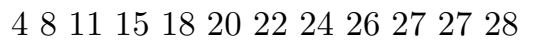 \\
\hline 79 & 4811151820222426272728 & 48121517202224252728 \\
\hline 80 & $\begin{array}{l}4710131618202224252627 \\
28\end{array}$ & $\begin{array}{l}\begin{array}{l}47 \\
28\end{array} \\
28\end{array}$ \\
\hline 81 & 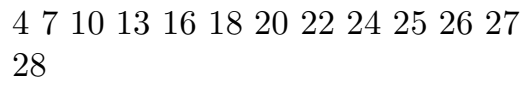 & $\begin{array}{l}\begin{array}{l}47 \\
28\end{array} 10131618202224252627 \\
\end{array}$ \\
\hline 82 & 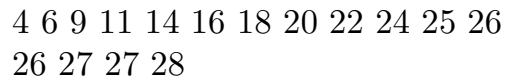 & 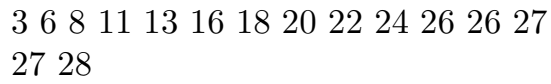 \\
\hline 83 & 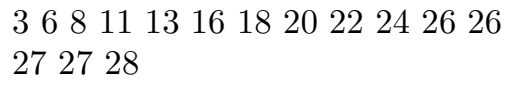 & $\begin{array}{l}46911141618202224252626 \\
272728\end{array}$ \\
\hline 84 & $\begin{array}{l}468101214161820212223 \\
2425262728\end{array}$ & $\begin{array}{l}46810121416182021222324 \\
25262728\end{array}$ \\
\hline 85 & 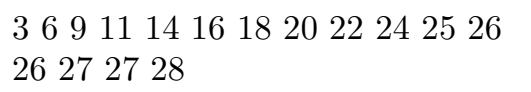 & 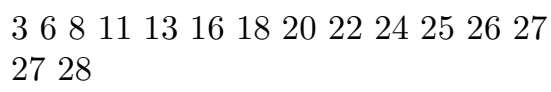 \\
\hline
\end{tabular}


TABLE 8. (continued)

\begin{tabular}{|c|c|c|}
\hline$k$ & $d_{0}(j, k) ; j=1,2, \ldots$ & $d_{1}(j, k) ; j=1,2, \ldots$ \\
\hline 86 & 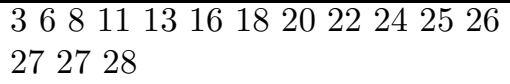 & 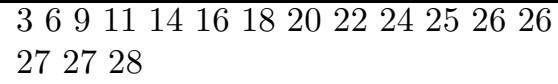 \\
\hline 87 & 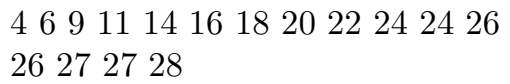 & $\begin{array}{l}26811131618202224262627 \\
2728\end{array}$ \\
\hline 88 & $\begin{array}{l}268111316182022242626 \\
272728\end{array}$ & $\begin{array}{l}46911141618202224242626 \\
272728\end{array}$ \\
\hline 89 & $\begin{array}{llllllllllll}3 & 5 & 8 & 10 & 12 & 14 & 16 & 18 & 19 & 21 & 22 & 23 \\
24 & 25 & 26 & 27 & 27 & 28\end{array}$ & $\begin{array}{l}25710121416182021222324 \\
25262728\end{array}$ \\
\hline 90 & 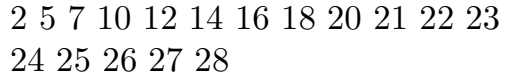 & $\begin{array}{l}35810121416181921222324 \\
2526272728\end{array}$ \\
\hline 91 & 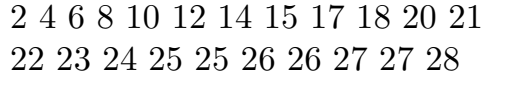 & $\begin{array}{l}24468101213151618192122 \\
2324252626272728\end{array}$ \\
\hline 92 & $\begin{array}{l}24681012131516181921 \\
222324252626272728\end{array}$ & $\begin{array}{l}246 \quad 6 \quad 101214151718202122 \\
232425252626272728\end{array}$ \\
\hline 93 & 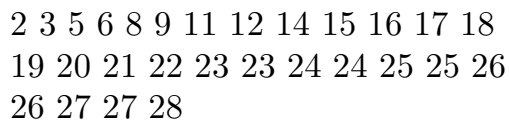 & $\begin{array}{l}13464791012131516171819 \\
202122232424252526262727 \\
28\end{array}$ \\
\hline 94 & 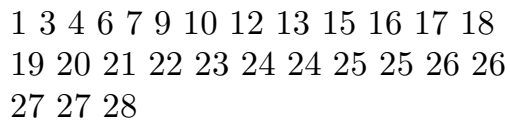 & 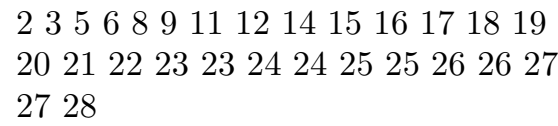 \\
\hline
\end{tabular}

\section{REFERENCES}

[1] D. Barbasch and M.R. Sepanski, Closure ordering and the Kostant-Sekiguchi correspondence, Proc. Amer. Math. Soc. 126 (1998), 311-317. MR 98c:22004

[2] W.M. Beynon and N. Spaltenstein, Green functions of finite Chevalley groups of type $E_{n}(n=$ 6, 7,8), J. Algebra 88 (1984), 584-614. MR 85k:20136

[3] N. Bourbaki, Groupes et algèbres de Lie, Chap. 7 et 8, Hermann, Paris, 1975. MR 56:12077

[4] B.W. Char, K.O. Geddes, G.H. Gonnet, B.L. Leong, M.B. Monagan, and S.M. Watt, Maple $V$ Language reference Manual, Springer-Verlag, New York, 1991, xv+267 pp.

[5] D.H. Collingwood and W.M. McGovern, Nilpotent Orbits in Semisimple Lie Algebras, Van Nostrand Reinhold, New York, 1993. MR 94j:17001

[6] D.Ž. Đoković, Classification of nilpotent elements in simple exceptional real Lie algebras of inner type and description of their centralizers, J. Algebra 112 (1988), 503-524. MR 89b:17010

[7] _ Explicit Cayley triples in real forms of $E_{7}$, Pacific J. Math. 191 (1999), 1-23. MR 2001c:22012

[8] _ The closure diagrams for nilpotent orbits of real forms of $F_{4}$ and $G_{2}$, J. Lie Theory 10 (2000), 491-510. MR 2001i:14061

[9] _ The closure diagrams for nilpotent orbits of real forms of $E_{6}$, J. Lie Theory 11 (2001), 381-413.

[10] The closure diagrams for nilpotent orbits of the real forms $E$ VI and EVII of $E_{7}$, Represent. Theory 5 (2001), 17-42. CMP 2001:11

[11] (to appear), $23 \mathrm{pp}$.

[12] K. Mizuno, The conjugate classes of unipotent elements of the Chevalley groups $E_{7}$ and $E_{8}$, Tokyo J. Math. 3 (1980), 391-461. MR 82m:20046

[13] M. Sato and T. Kimura, A classification of irreducible prehomogeneous vector spaces and their relative invariants, Nagoya Math. J. 65 (1977), 1-155. MR 55:3341 
[14] M. Sato, Theory of prehomogeneous vector spaces (algebraic part), the English translation of Sato's lecture from Shintani's notes. Notes by Takuro Shintani. Translated from the Japanese by Masakazu Muro, Nagoya Math. J. 120 (1990), 1-34. MR 92c:32039

[15] J. Tits, Normalisateurs de tores. I. Groupes de Coxeter étendus, J. Algebra 4 (1966), 96-116. MR 34:5942

[16] M.A.A. van Leeuwen, A.M. Cohen, and B. Lisser, "LiE", a software package for Lie group theoretic computations, Computer Algebra Group of CWI, Amsterdam, The Netherlands.

Department of Pure Mathematics, University of Waterloo, Waterloo, Ontario, N2L 3G1, CANADA

E-mail address: djokovic@uwaterloo.ca 